COMMUNICATIONS IN

ANALYSIS AND GEOMETRY

Volume 3, Number 3, 421-463, 1995

\title{
Higher cohomology triples and holomorphic extensions
}

\begin{abstract}
Steven B. Bradlow and Oscar García-Prada
We introduce equations for special metrics, and notions of stability for some new types of augmented holomorphic bundles. These new examples include holomorphic extensions, and in this case we prove a Hitchin-Kobayashi correspondence between a certain deformation of the Hermitian-Einstein equations and our definition of stability for an extension.
\end{abstract}

\section{Introduction.}

There are three natural moduli spaces associated to a smooth complex bundle over a Kähler manifold; one algebraic, one complex analytic, and one symplectic. The first is the moduli space of slope stable holomorphic structures on $E$, and is constructed by Geometric Invariant Theory. The second, the moduli space of Hermitian-Einstein connections, is constructed by gauge theory and deformation theory. For the third, one uses the symplectic structure induced on the space of unitary connections and considers the moment map for the action of the unitary gauge group. The symplectic moduli space is then the Marsden-Weinstein quotient of the zero level of the moment map by the action of the unitary gauge group.

In fact, it is well known that these three quotients can all be identified, and this is referred to as the Hitchin-Kobayashi correspondence. Furthermore, this triad of descriptions has, in recent years, been found to be a common feature in an ever expanding range of situations. In most of these, the moduli spaces are for augmented bundles of one kind or another, i.e. for objects consisting of one or more holomorphic bundle together with prescribed holomorphic sections. A summary of such results can be found in [BDGW].

In this paper we discuss some extensions of these ideas in two directions that have not hitherto been pursued. This involves consideration of an interesting class of equations which includes deformations of the HermitianEinstein equations as well as certain generalizations of the equations known 
as the vortex equations. It also requires the introduction of a new concepts of stability for various augmented bundles.

In the one class of examples that we discuss, the starting point is the observation that symplectic reduction can be carried out more generally than simply at the 0-level set. In particular, symplectic quotients can be constructed from the inverse images of coadjoint orbits in the dual of the Lie algebra of the unitary gauge group. It is natural to look for a description of such reduced spaces as complex quotients and to try to find an algebraic characterization of these quotients as moduli spaces.

The simplest example of such a generalization can be described as follows. Suppose that $E=E_{1} \oplus E_{2}$. Fix a smooth metric $K$ on $E$ such that the above splitting of $E$ is an orthogonal decomposition. Let $T_{\tau_{1}, \tau_{2}} \in \mathfrak{G}$ be the global gauge transformation given by

$$
T_{\tau_{1}, \tau_{2}}=\left(\begin{array}{cc}
i \tau_{1} \mathbf{I}_{1} & 0 \\
0 & i \tau_{2} \mathbf{I}_{2}
\end{array}\right)
$$

with respect to the given splitting of $E$, and let $\mathcal{O}\left(\tau_{1}, \tau_{2}\right)$ be the coadjoint orbit of $T_{\tau_{1}, \tau_{2}}$. Pursuing this example, we find interesting "deformations" of both the Hermitian-Einstein equations and the notion of bundle stability. Furthermore, these are naturally interpreted in terms of holomorphic extensions.

The second type of structure we consider is a natural generalization of the triples described in [BGP]. In [BGP] we described objects consisting of two holomorphic bundles, $\mathcal{E}_{1}$ and $\mathcal{E}_{2}$ plus a map between them, i.e. a section $\Phi \in H^{0}\left(X, \operatorname{Hom}\left(\mathcal{E}_{2}, \mathcal{E}_{1}\right)\right)$. In the generalization we have in mind, we take $\Phi$ in $H^{p}\left(X, \operatorname{Hom}\left(\mathcal{E}_{2}, \mathcal{E}_{1}\right)\right)$, for any $p$. We call such objects $p$-cohomology triples. Apart from their interest as natural generalizations of the original triples, such objects (with $p=2$ ) have been encountered in the work of Pidstrigach and Tyurin ([PT]), and more recently in connection with the Seiberg-Witten invariants for Kähler surfaces (cf. [W]). For the case $p=0$, we described in [BGP] what the natural notion of stability is, and what the corresponding equations for special metrics look like. In this paper we discuss how these can be modified to describe the more general situation.

The case of $p=1$ is of particular interest, since elements in

$$
H^{1}\left(X, \operatorname{Hom}\left(\mathcal{E}_{2}, \mathcal{E}_{1}\right)\right)
$$

can be interpreted as extension classes. This leads to interesting relations between the two kinds of situations described above. We describe in some detail how these points of view compare. We also relate these to yet another 
description of holomorphic extensions, namely one in terms of the bundles $\mathcal{E}, \mathcal{E}_{2}$ plus surjective maps $\pi: \mathcal{E} \longrightarrow \mathcal{E}_{2}$. Such objects, which describe extensions of $\mathcal{E}_{2}$ by the kernel of the map, can be thought of as a special type of $p=0$ triples. More specifically, they correspond to such triples in which the map between the bundles is surjective. We thus discuss the relation between such surjective $(p=0)$ triples, 1-cohomology triples and extensions.

Remark. The result given in Theorem 3.9 has been proved independently by Daskalopoulos, Uhlenbeck and Wentworth [DUW]. With stability defined as in in Definition 3.4, they have gone on to give analytic as well as invariant theory constructions of the moduli spaces of stable extensions.

\section{Acknowledgements.}

The authors would like to thank Alastair King for many helpful conversations and ideas, especially with regard to the formulation of the definitions of stability in $§ 2.2$. The authors would also like to thank Fiammetta Battaglia for useful conversations. Both authors are members of the VBAC group of Europroj. The first author was partially supported by NSF grant DMS 93-03545. The work of the second author was partially carried out at the Mathematics Department of the University of Paris-Sud with the support of a European Union postdoctoral fellowship under the HCM programme.

\section{Cohomology triples.}

Let $(X, \omega)$ be a compact Kähler manifold of dimension $n$, and fix two smooth complex bundles $E_{i} \longrightarrow X, i=1,2$. Denote their ranks and degrees by $d_{i}$ and $r_{i}$, where by the degree we mean, in general, $\int_{X} c_{1}\left(E_{1}\right) \wedge \omega^{n-1}$. In order to simplify certain formulae, we assume that the volume of $X$ is normalised to $2 \pi$.

As defined in [GP] and [BGP], a holomorphic triple based on $E_{1}$ and $E_{2}$ consists of holomorphic structures (given by $\bar{\partial}$-operators $\bar{\partial}_{1}$ and $\bar{\partial}_{2}$ ) on these bundles plus a holomorphic section of $\operatorname{Hom}\left(E_{2}, E_{1}\right)$.

There are two distinct ways in which one might want to generalize this to allow form-valued augmentations.

1. In the first, which we call p-cocycle triples, one replaces holomorphic sections in $\Omega^{0}\left(\operatorname{Hom}\left(E_{2}, E_{1}\right)\right)$ by holomorphic sections in $\Omega^{0, p}\left(\operatorname{Hom}\left(E_{2}, E_{1}\right)\right)$. 
2. In the second, which we call p-cohomology triples, the augmentation is considered to be the class in $H^{0, p}\left(H o m\left(E_{2}, E_{1}\right)\right)$ represented by a holomorphic section in $\Omega^{0, p}\left(\operatorname{Hom}\left(E_{2}, E_{1}\right)\right)$.

As will be seen (cf. Section 2.2 ), there are compelling reasons for regarding the second approach as the "correct" one. Nevertheless, at least in the case where $X$ is a Riemann surface, there are interesting features of both types of augmentation. In the case that $\mathcal{E}_{2}$ is fixed to be the structure sheaf, the resulting objects may be considered as $p$-cocycle and $p$-cohomology pairs.

\subsection{The basics.}

Set

$$
\chi^{(p)}=\mathcal{C}_{1} \times \mathcal{C}_{2} \times \Omega^{0, p}\left(\operatorname{Hom}\left(E_{2}, E_{1}\right)\right),
$$

where $\mathcal{C}_{i}$ denotes the space of holomorphic structures (or equivalently, the space of $\bar{\partial}$-operators) on $E_{i}$.

Definition 2.1. We can define the space of all p-cocycle triples on $\left(E_{1}, E_{2}\right)$ by the holomorphic subspace

$$
\mathcal{Z}^{(p)}=\left\{\left(\bar{\partial}_{1}, \bar{\partial}_{2}, \phi\right) \in \chi^{(p)}: \bar{\partial}_{1,2}(\phi)=0\right\},
$$

where $\bar{\partial}_{1,2}(\phi)=\bar{\partial}_{1} \circ \phi-\phi \circ \bar{\partial}_{2}$.

We can define an equivalence relation on $\mathcal{Z}^{(p)}$ by

$$
\left(\bar{\partial}_{1}, \bar{\partial}_{2}, \phi\right) \sim\left(\bar{\partial}_{1}, \bar{\partial}_{2}, \phi+\bar{\partial}_{1,2}(\alpha)\right),
$$

for any $\alpha \in \Omega^{0, p-1}\left(\operatorname{Hom}\left(E_{2}, E_{1}\right)\right)$. The $p$-cohomology triples are described by the equivalence classes in $\mathcal{Z}^{(p)} / \sim$. Notice that these equivalence classes correspond to orbits of the additive group $\Omega_{1,2}^{p-1}:=\Omega^{0, p-1}\left(\operatorname{Hom}\left(E_{2}, E_{1}\right)\right)$ under the action

$$
\alpha \circ\left(\bar{\partial}_{1}, \bar{\partial}_{2}, \phi\right)=\left(\bar{\partial}_{1}, \bar{\partial}_{2}, \phi+\bar{\partial}_{1,2}(\alpha)\right) .
$$

Definition 2.2. The space of all p-cohomology triples on $\left(E_{1}, E_{2}\right)$ is defined by

$$
\mathcal{H}^{(p)}=\mathcal{Z}^{(p)} / \Omega_{1,2}^{p-1}
$$


Definition/Lemma 2.3. The complex gauge group $\mathfrak{G}_{\mathbb{C}}=\mathfrak{G}_{\mathbb{C}}^{(1)} \times \mathfrak{G}_{\mathbb{C}}^{(2)}$ acts on both $\mathcal{Z}^{(p)}$ and $\mathcal{H}^{(p)}$. In both cases, the $\mathfrak{G}_{\mathbb{C}^{-} \text {orbits correspond to iso- }}$ morphism classes, with the notion of isomorphism defined in the obvious way. Thus the "moduli spaces" of isomorphism classes of $p$-cocycle (resp. $p$-cohomology) triples corresponds to the orbit space $\mathcal{Z}^{(p)} / \mathfrak{G}_{\mathbb{C}}$ (resp. $\left.\mathcal{H}^{(p)} / \mathfrak{G}_{\mathbb{C}}\right)$

It is important to observe that in the double quotient

$$
\mathcal{H}^{(p)} / \mathfrak{G}_{\mathbb{C}}=\left(\mathcal{Z}^{(p)} / \Omega_{1,2}^{p-1}\right) / \mathfrak{G}_{\mathbb{C}},
$$

the order of the quotient operations cannot be reversed. Not only do the actions of $\Omega_{1,2}^{p-1}$ and $\mathfrak{G}_{\mathbb{C}}$ fail to commute, but $\Omega_{1,2}^{p-1}$ does not act on $\mathcal{Z}^{(p)} / \mathfrak{G}_{\mathbb{C}}$ in any obvious way. Nevertheless, the quotient $\mathcal{H}^{(p)} / \mathfrak{G}_{\mathbb{C}}$ can be described as a quotient of $\mathcal{Z}^{(p)}$, namely as the quotient by the group action of the semidirect product $\Omega_{1,2}^{p-1} \ltimes \mathfrak{G}_{\mathbb{C}}$.

Definition/Lemma 2.4. We can identify

$$
\left(\mathcal{Z}^{(p)} / \Omega_{1,2}^{p-1}\right) / \mathfrak{G}_{\mathbb{C}}=\mathcal{H}^{(p)} / \Omega_{1,2}^{p-1} \ltimes \mathfrak{G}_{\mathbb{C}}
$$

where the group structure on the semidirect product is defined by

$$
\left(\alpha, g_{1}, g_{2}\right)\left(\alpha^{\prime}, g_{1}^{\prime}, g_{2}^{\prime}\right)=\left(g_{1}^{\prime-1} \alpha g_{2}^{\prime}+\alpha^{\prime}, g_{1} g_{1}^{\prime}, g_{2} g_{2}^{\prime}\right)
$$

and the action on $\mathcal{Z}^{(p)}$ is

$$
\left(\alpha, g_{1}, g_{2}\right)\left(\bar{\partial}_{1}, \bar{\partial}_{2}, \phi\right)=\left(g_{1}\left(\bar{\partial}_{1}\right), g_{2}\left(\bar{\partial}_{2}\right), g_{1}\left(\phi+\bar{\partial}_{1,2}(\alpha)\right) g_{2}^{-1}\right)
$$

\subsection{Stability with parameters.}

As usual, one cannot expect the orbit spaces $\mathcal{Z}^{(p)} / \mathfrak{G}_{\mathbb{C}}$ or $\mathcal{H}^{(p)} / \mathfrak{G}_{\mathbb{C}}$ to yield well behaved moduli spaces without restricting to suitably defined spaces of "stable" orbits. The definition of stability that we propose for cohomology triples is a reasonably straightforward extensions of the stability defined for triples in [BGP]. Since this definition is in terms of a condition on subtriples, we need to specify precisely what we mean by the subobjects of cohomology triples.

Let $\mathcal{E}_{1}=\left(E_{1}, \bar{\partial}_{1}\right)$ and $\mathcal{E}_{2}=\left(E_{2}, \bar{\partial}_{2}\right)$ holomorphic vector bundles on $X$ and $\Phi \in H^{p}\left(\operatorname{Hom}\left(\mathcal{E}_{2}, \mathcal{E}_{1}\right)\right)$. To define the subobjects of the cohomology triple $T=\left(\mathcal{E}_{1}, \mathcal{E}_{2}, \Phi\right)$ we need to determine the category to which $T$ belongs. 
The subobjects of $T$ will be then certain objects in this category-the ones for which there is an injective morphism to $T$.

The category we need to consider is the category of " $\mathrm{Ext}^{p}$ " triples. Its elements consist of triples $\left(\mathcal{F}_{1}, \mathcal{F}_{2}, \Psi\right)$, where $\mathcal{F}_{1}$ and $\mathcal{F}_{2}$ are coherent sheaves on $X$ and $\Psi$ is an element of $\operatorname{Ext}^{p}\left(\mathcal{F}_{2}, \mathcal{F}_{1}\right)$.

Recall that $\operatorname{Ext}^{0}\left(\mathcal{F}_{2}, \mathcal{F}_{1}\right)=\operatorname{Hom}\left(\mathcal{F}_{2}, \mathcal{F}_{1}\right) \cong H^{0}\left(\operatorname{Hom}\left(\mathcal{F}_{2}, \mathcal{F}_{1}\right)\right)$, and if $\mathcal{F}_{2}$ is locally free:

1) $\operatorname{Hom}\left(\mathcal{F}_{2}, \mathcal{F}_{1}\right) \cong \mathcal{F}_{1} \otimes \mathcal{F}_{2}^{*}$

2) $\operatorname{Ext}^{p}\left(\mathcal{F}_{2}, \mathcal{F}_{1}\right) \cong H^{p}\left(\mathcal{F}_{1} \otimes \mathcal{F}_{2}^{*}\right)$.

Let $T=\left(\mathcal{F}_{1}, \mathcal{F}_{2}, \Psi\right)$ and $T^{\prime}=\left(\mathcal{F}_{1}^{\prime}, \mathcal{F}_{2}^{\prime}, \Psi^{\prime}\right)$ be two Ext triples. A morphism $T^{\prime} \longrightarrow T$ consists of morphisms $f_{1}: \mathcal{F}_{1}^{\prime} \longrightarrow \mathcal{F}_{1}$ and $f_{2}: \mathcal{F}_{2}^{\prime} \longrightarrow \mathcal{F}_{2}$ such that under the induced maps

$$
\operatorname{Ext}^{p}\left(\mathcal{F}_{2}^{\prime}, \mathcal{F}_{1}^{\prime}\right) \stackrel{f_{1 *}}{\longrightarrow} \operatorname{Ext}^{p}\left(\mathcal{F}_{2}^{\prime}, \mathcal{F}_{1}\right) \stackrel{f_{2}^{*}}{\longleftarrow} \operatorname{Ext}^{p}\left(\mathcal{F}_{2}, \mathcal{F}_{1}\right)
$$

one has that

$$
f_{1 *}\left(\Psi^{\prime}\right)=f_{2}^{*}(\Psi)
$$

Note that when $p=0$, this is equivalent to having the following commutative diagram

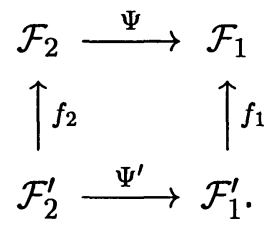

Definition 2.5. Let $T=\left(\mathcal{F}_{1}, \mathcal{F}_{2}, \Psi\right)$ be an Ext ${ }^{p}$ triple. A subobject $T^{\prime}$ of $T$ consists of an Ext ${ }^{p}$ triple $\left(\mathcal{F}_{1}^{\prime}, \mathcal{F}_{2}^{\prime}, \Psi^{\prime}\right)$ such that one has injections $i_{1}: \mathcal{F}_{1}^{\prime} \hookrightarrow \mathcal{F}_{1}$ and $i_{2}: \mathcal{F}_{2}^{\prime} \hookrightarrow \mathcal{F}_{2}$, which induce a morphism from $T^{\prime}$ to $T$, i.e. $i_{1 *}\left(\Psi^{\prime}\right)=i_{2}^{*}(\Psi)$.

Notice that if $\left(\mathcal{E}_{1}, \mathcal{E}_{2}, \Phi\right)$ is a cohomology triple, (where $\mathcal{E}_{1}$ and $\mathcal{E}_{2}$ are locally free and $\left.\Phi \in H^{p}\left(\operatorname{Hom}\left(\mathcal{E}_{2}, \mathcal{E}_{1}\right)\right)\right)$, a subobject need not be a cohomology triple. In general it will be only an $\mathrm{Ext}^{p}$ triple, i.e. $\mathcal{E}_{1}^{\prime} \hookrightarrow \mathcal{E}_{1}$ and $\mathcal{E}_{2}^{\prime} \hookrightarrow \mathcal{E}_{2}$ are not necessarily locally free and $\Phi^{\prime} \in \operatorname{Ext}^{p}\left(\mathcal{E}_{2}^{\prime}, \mathcal{E}_{1}^{\prime}\right)$ (If $\mathcal{E}_{2}^{\prime}$ is not locally free this cannot be identified with $H^{p}\left(\operatorname{Hom}\left(\mathcal{E}_{2}^{\prime}, \mathcal{E}_{1}^{\prime}\right)\right)$.

The definition of stability is given in terms of defect functions (Alastair King's terminology) for pairs of bundles: 
Definition 2.6. Let $\left(E_{1}, E_{2}\right)$ be a pair of bundles of degree $d_{1}$ and $d_{2}$, and $\operatorname{rank} r_{1}$ and $r_{2}$. Fix real numbers $\left\{a_{1}, a_{2}, \tau_{1}, \tau_{2}\right\}$, and define $\theta_{a_{1}, a_{2}, \tau_{1}, \tau_{2}}\left(\mathcal{E}_{1}, \mathcal{E}_{2}\right)$ by

$$
\theta_{a_{1}, a_{2}, \tau_{1}, \tau_{2}}\left(\mathcal{E}_{1}, \mathcal{E}_{2}\right)=a_{1} d_{1}+a_{2} d_{2}-\tau_{1} r_{1}-\tau_{2} r_{2} .
$$

Of course this definition makes also sense if $\mathcal{E}_{1}$ and $\mathcal{E}_{2}$ are coherent sheaves not necessarily locally free.

Definition 2.7. Let $\left(\mathcal{E}_{1}, \mathcal{E}_{2}, \Phi\right)$ be a $p$-cohomology triple based on the smooth bundles $\left(E_{1}, E_{2}\right)$. Fix real numbers $\left\{a_{1}, a_{2}, \tau_{1}, \tau_{2}\right\}$ with $a_{1}$ and $a_{2}$ non-negative, and such that

$$
a_{1} d_{1}+a_{2} d_{2}-\tau_{1} r_{1}-\tau_{2} r_{2}=0,
$$

i.e. such that $\theta_{a_{1}, a_{2}, \tau_{1}, \tau_{2}}\left(\mathcal{E}_{1}, \mathcal{E}_{2}\right)=0$. We say that the triple $\left(\mathcal{E}_{1}, \mathcal{E}_{2}, \Phi\right)$ is $\left(a_{1}, a_{2}, \tau_{1}, \tau_{2}\right)$-stable if

$$
\theta_{a_{1}, a_{2}, \tau_{1}, \tau_{2}}\left(\mathcal{E}_{1}^{\prime}, \mathcal{E}_{2}^{\prime}\right)<0
$$

for all non-trivial subobjects $\left(\mathcal{E}_{1}^{\prime}, \mathcal{E}_{2}^{\prime}, \Phi^{\prime}\right)$.

\section{Remarks.}

1. As usual, to study stability questions it suffices to consider saturated subobjects of $\left(\mathcal{E}_{1}, \mathcal{E}_{2}, \Phi\right)$ (we are assuming that $\mathcal{E}_{1}$ and $\mathcal{E}_{2}$ are torsion free). These are subobjects $\left(\mathcal{E}_{1}^{\prime}, \mathcal{E}_{2}^{\prime}, \Phi^{\prime}\right)$ for which the inclusions $\mathcal{E}_{1}^{\prime} \hookrightarrow$ $\mathcal{E}_{1}$ and $\mathcal{E}_{2}^{\prime} \hookrightarrow \mathcal{E}_{2}$ are saturated, i.e. $\mathcal{E}_{1} / \mathcal{E}_{1}^{\prime}$ and $\mathcal{E}_{2} / \mathcal{E}_{2}^{\prime}$ are torsion free.

2. As in the case of cohomology triples, before we can define stability for a cocycle triple, we must first establish what the legitimate subobjects are. We immediately run into difficulty when we consider what the appropriate category for such objects should be. Denote the objects in the category by $\left(\mathcal{F}_{1}, \mathcal{F}_{2}, \phi\right)$. The problem is that we do not want to require that $\mathcal{F}_{1}$ and $\mathcal{F}_{2}$ be locally free sheaves. This means we need to have a replacement for $\Omega^{0, p}\left(\operatorname{Hom}\left(\mathcal{F}_{2}, \mathcal{F}_{1}\right)\right)$ in the case that $\mathcal{F}_{1}$ and $\mathcal{F}_{2}$ are not locally free. This is one of the reasons that cohomology triples are to be preferred to their cocycle cousins .

Notice that when $X$ is a Riemann surface, these difficulties do not arise, and sensible definitions can be given. Subobjects are defined to be cocycle triples $\left(\mathcal{F}_{1}^{\prime}, \mathcal{F}_{2}^{\prime}, \phi^{\prime}\right)$ with injections $i_{1}: \mathcal{F}_{1}^{\prime} \longrightarrow \mathcal{F}_{1}$ and $i_{2}: \mathcal{F}_{2}^{\prime} \longrightarrow \mathcal{F}_{2}$ such that

$$
i_{1} \circ \phi^{\prime}=\phi^{\prime} \circ i_{2} .
$$


Stability with respect to parameters $\left\{a_{1}, a_{2}, \tau_{1}, \tau_{2}\right\}$ is then defined exactly as for cohomology triples.

3. Finally, suppose that $\left(\mathcal{E}_{1}, \mathcal{E}_{2}, \phi\right)$ is a 0-cohomology triple, i.e. a triple with $\phi \in H^{0}\left(\operatorname{Hom}\left(E_{2}, E_{1}\right)\right)$. We recover the old definition of $\tau$ stability given in [BGP] by taking $\left\{a_{1}, a_{2}, \tau_{1}, \tau_{2}\right\}=\left\{1,1, \tau, \tau^{\prime}\right\}$. The definition above is thus a generalization of $\tau$-stability.

The parameter space for the parameters in the definition of stability can be described as follows. Let Par $\subset \mathbb{R}^{4}$ be the subspace (2.2.3)

$$
\operatorname{Par}=\left\{\left(a_{1}, a_{2}, \tau_{1}, \tau_{2}\right) \mid a_{1} \geq 0, a_{2} \geq 0, a_{1} d_{1}+a_{2} d_{2}-\tau_{1} r_{1}-\tau_{2} r_{2}=0\right\} .
$$

Notice that the definition of $\left(a_{1}, a_{2}, \tau_{1}, \tau_{2}\right)$-stability is insensitive to an overall scaling of $\left(a_{1}, a_{2}, \tau_{1}, \tau_{2}\right)$ by a positive scale factor. The effective parameter space is thus Par $/ \mathbb{R}^{+}$. The "geography" of this parameter space is an interesting issue, which we will return to in a later paper. There are however, a few features which are immediately apparent.

The first feature comes from the fact that (at least for the case when $X$ is algebraic), the degrees and ranks of subobjects may be assumed to be integers, i.e. to lie in a discrete subset of $\mathbb{R}$. It follows immediately that

Lemma 2.8. The parameter space $\mathrm{Par} / \mathbb{R}^{+}$is partitioned into chambers. The walls are determined by the choices of $\left(a_{1}, a_{2}, \tau_{1}, \tau_{2}\right)$ at which the relation $\theta_{a_{1}, a_{2}, \tau_{1}, \tau_{2}}\left(\mathcal{E}_{1}^{\prime}, \mathcal{E}_{2}^{\prime}\right)=0$ is numerically possible. Within a fixed chamber the definition of $\left(a_{1}, a_{2}, \tau_{1}, \tau_{2}\right)$-stability is independent of the values of $\left(a_{1}, a_{2}, \tau_{1}, \tau_{2}\right)$.

The next result identifies a special region within $\operatorname{Par} / \mathbb{R}^{+}$.

Proposition 2.9. (1) Suppose that $a_{1}>0$. Then the space of $\left(a_{1}, a_{2}, \tau_{1}, \tau_{2}\right)$-stable objects is empty unless $\tau_{1} / a_{1}>\mu\left(\mathcal{E}_{1}\right)$.

(2) There are positive numbers $\epsilon_{1}, \epsilon_{2}$ such that the following is true: Let $\left(a_{1}, a_{2}, \tau_{1}, \tau_{2}\right)$ be any point in Par such that

$$
\begin{aligned}
& a_{1} a_{2} \neq 0, \\
& 0<\frac{\tau_{1}}{a_{1}}-\mu\left(E_{1}\right)<\epsilon_{1}, \\
& \frac{\tau_{1}}{a_{1}}-\mu\left(E_{1}\right)<\left(\frac{a_{2}}{a_{1}}\right) \epsilon_{2} .
\end{aligned}
$$

Then in any $\left(a_{1}, a_{2}, \tau_{1}, \tau_{2}\right)$-stable object, say $\left(\mathcal{E}_{1}, \mathcal{E}_{2}, \Phi\right)$, the bundles $\mathcal{E}_{1}$ and $\mathcal{E}_{2}$ are semistable. Conversely, if $\mathcal{E}_{1}$ and $\mathcal{E}_{2}$ are stable bundles, then all cohomology triples $\left(\mathcal{E}_{1}, \mathcal{E}_{2}, \Phi\right)$ are $\left(a_{1}, a_{2}, \tau_{1}, \tau_{2}\right)$-stable. 
Proof. Both parts of the proposition use the following observations. Let $\left(\mathcal{E}_{1}, \mathcal{E}_{2}, \Phi\right)$ be any $p$-cohomology triple. For any subsheaf $\mathcal{E}_{1}^{\prime}$ of $\mathcal{E}_{1}$ we can construct the subtriple $\left(\mathcal{E}_{1}^{\prime}, 0,0\right)$. Furthermore, for any subsheaf $\mathcal{E}_{2}^{\prime} \hookrightarrow \mathcal{E}_{2}$ we can construct the subtriple $\left(\mathcal{E}_{1}, \mathcal{E}_{2}^{\prime}, \Phi^{\prime}\right)$ by taking $\Phi^{\prime}=i_{2}^{*}(\Phi)$. Notice that

$$
\theta_{a_{1}, a_{2}, \tau_{1}, \tau_{2}}\left(\mathcal{E}_{1}^{\prime}, 0\right)=a_{1} r_{1}^{\prime}\left(\mu\left(\mathcal{E}_{1}^{\prime}\right)-\tau_{1} / a_{1}\right) .
$$

Part (1) follows immediately from this. For part (2), we observe that if $a_{1} \neq 0$, then we can write (2.2.4) as

$$
\frac{\theta_{a_{1}, a_{2}, \tau_{1}, \tau_{2}}\left(\mathcal{E}_{1}^{\prime}, 0\right)}{a_{1}}=r_{1}^{\prime}\left(\mu\left(\mathcal{E}_{1}^{\prime}\right)-\mu\left(\mathcal{E}_{1}\right)\right)+r_{1}^{\prime}\left(\mu\left(\mathcal{E}_{1}\right)-\tau_{1} / a_{1}\right) .
$$

It follows that if $\left|\mu\left(\mathcal{E}_{1}\right)-\tau_{1} / a_{1}\right|$ is sufficiently small, and if $\theta_{a_{1}, a_{2}, \tau_{1}, \tau_{2}}\left(\mathcal{E}_{1}^{\prime}, 0\right)<0$, then $\mu\left(\mathcal{E}_{1}^{\prime}\right)-\mu\left(\mathcal{E}_{1}\right) \leq 0$. We now consider the subobjects coming from subsheaves of $\mathcal{E}_{2}$. For these,we get

$$
\theta_{a_{1}, a_{2}, \tau_{1}, \tau_{2}}\left(\mathcal{E}_{1}, \mathcal{E}_{2}^{\prime}\right)=a_{1} d_{1}+a_{2} d_{2}^{\prime}-r_{1} \tau_{1}-r_{2}^{\prime} \tau_{2} .
$$

Using the constraint equation $a_{1} d_{1}+a_{2} d_{2}-\tau_{1} r_{1}-\tau_{2} r_{2}=0$, this can be written as

$$
\frac{\theta_{a_{1}, a_{2}, \tau_{1}, \tau_{2}}\left(\mathcal{E}_{1}, \mathcal{E}_{2}^{\prime}\right)}{a_{2}}=r_{2}^{\prime}\left(\mu\left(\mathcal{E}_{2}^{\prime}\right)-\mu\left(\mathcal{E}_{2}\right)\right)-r_{1}\left(\frac{r_{2}-r_{2}^{\prime}}{r_{2}}\right)\left(\frac{\tau_{1} / a_{1}-\mu\left(\mathcal{E}_{1}\right)}{a_{2} / a_{1}}\right) .
$$

Thus if $\theta_{a_{1}, a_{2}, \tau_{1}, \tau_{2}}\left(\mathcal{E}_{1}, \mathcal{E}_{2}^{\prime}\right)<0$, then

$$
\mu\left(\mathcal{E}_{2}^{\prime}\right)-\mu\left(\mathcal{E}_{2}\right)<\left(\frac{r_{1}}{r_{2}^{\prime}}\right)\left(\frac{r_{2}-r_{2}^{\prime}}{r_{2}}\right)\left(\frac{\tau_{1} / a_{1}-\mu\left(\mathcal{E}_{1}\right)}{a_{2} / a_{1}}\right) .
$$

Since $\left(\frac{r_{1}}{r_{2}^{\prime}}\right)\left(\frac{r_{2}-r_{2}^{\prime}}{r_{2}}\right)$ is bounded above, it follows that $\mu\left(\mathcal{E}_{2}^{\prime}\right)-\mu\left(\mathcal{E}_{2}\right) \leq 0$ if $\frac{\tau_{1} / a_{1}-\mu\left(\mathcal{E}_{1}\right)}{a_{2} / a_{1}}$ is sufficiently small. This completes the proof of the first claim in (2). The second claim also follows from the identities (2.2.5) and (2.2.7), which show that for any subtriple $\left(\mathcal{E}_{1}, \mathcal{E}_{2}^{\prime}, \Phi^{\prime}\right)$ we have

$$
\begin{aligned}
& \theta_{a_{1}, a_{2}, \tau_{1}, \tau_{2}}\left(\mathcal{E}_{1}^{\prime}, \mathcal{E}_{2}^{\prime}\right)=\theta_{a_{1}, a_{2}, \tau_{1}, \tau_{2}}\left(\mathcal{E}_{1}, \mathcal{E}_{2}^{\prime}\right)+\theta_{a_{1}, a_{2}, \tau_{1}, \tau_{2}}\left(\mathcal{E}_{1}^{\prime}, 0\right)-a_{1} r_{1}\left(\mu\left(\mathcal{E}_{1}\right)-\tau_{1} / a_{1}\right) \\
= & a_{2} r_{2}^{\prime}\left(\mu\left(\mathcal{E}_{2}^{\prime}\right)-\mu\left(\mathcal{E}_{2}\right)\right)+a_{1} r_{1}^{\prime}\left(\mu\left(\mathcal{E}_{1}^{\prime}\right)-\mu\left(\mathcal{E}_{1}\right)\right)+a_{1}\left(r_{1}-r_{1}^{\prime}\right)\left(\tau_{1} / a_{1}-\mu\left(\mathcal{E}_{1}\right)\right) \\
- & a_{2} r_{1}\left(\frac{r_{2}-r_{2}^{\prime}}{r_{2}}\right)\left(\frac{\tau_{1} / a_{1}-\mu\left(\mathcal{E}_{1}\right)}{a_{2} / a_{1}}\right) .
\end{aligned}
$$




\subsection{Comparison of cocycle and cohomology.}

In the case that $X$ is a Riemann surface, the following comparison between cohomology and cocycle triples makes sense. Let $\pi: \mathcal{Z}^{(p)} \longrightarrow \mathcal{H}^{(p)}=$ $\mathcal{Z}^{(p)} / \Omega_{1,2}^{p-1}$ denote the projection map. Let $\left(a_{1}, a_{2}, \tau_{1}, \tau_{2}\right)$ be any set of real numbers satisfying the constraint

$$
a_{1} d_{1}+a_{2} d_{2}-\tau_{1} r_{1}-\tau_{2} r_{2}=0 .
$$

Proposition 2.10. Suppose that $X$ is a Riemann surface. For any cohomology triple $\left(\bar{\partial}_{1}, \bar{\partial}_{2}, \Phi\right) \in \mathcal{H}^{(p)}$, the following are equivalent:

1. $\left(\bar{\partial}_{1}, \bar{\partial}_{2}, \Phi\right) \in \mathcal{H}^{(p)}$ is $\left(a_{1}, a_{2}, \tau_{1}, \tau_{2}\right)$-stable,

2. all cocycle triples $\left(\bar{\partial}_{1}, \bar{\partial}_{2}, \phi\right) \in \pi^{-1}\left(\bar{\partial}_{1}, \bar{\partial}_{2}, \Phi\right)$ are $\left(a_{1}, a_{2}, \tau_{1}, \tau_{2}\right)$-stable,

3. given any $\left(\bar{\partial}_{1}, \bar{\partial}_{2}, \phi\right) \in \pi^{-1}\left(\bar{\partial}_{1}, \bar{\partial}_{2}, \Phi\right)$, every cocycle triple on the $\Omega_{1,2}^{p-1}$ orbit through $\left(\bar{\partial}_{1}, \bar{\partial}_{2}, \phi\right)$ is $\left(a_{1}, a_{2}, \tau_{1}, \tau_{2}\right)$-stable.

Proof. Statements (2) and (3) are obviously equivalent. We thus need only prove that (2) or (3) is equivalent to (1). To do so, we need to compare the definitions of stability for a cocycle triple and for a cohomology triple. In both cases, the definition is given in terms of the values of $\theta_{a_{1}, a_{2}, \tau_{1}, \tau_{2}}\left(\mathcal{E}_{1}^{\prime}, \mathcal{E}_{2}^{\prime}\right)$, where $\mathcal{E}_{1}^{\prime}$ and $\mathcal{E}_{2}^{\prime}$ are the subbundles in either a cocycle subtriple, $\left(\bar{\partial}_{1}^{\prime}, \bar{\partial}_{2}^{\prime}, \phi^{\prime}\right)$, or a cohomology subtriple, $\left(\bar{\partial}_{1}^{\prime}, \bar{\partial}_{2}^{\prime}, \Phi^{\prime}\right)$. Notice that neither the $\phi^{\prime}$ nor the $\Phi^{\prime}$ affect the value of $\theta_{a_{1}, a_{2}, \tau_{1}, \tau_{2}}$-their only role is to determine on which pairs $\left(\mathcal{E}_{1}^{\prime}, \mathcal{E}_{2}^{\prime}\right)$ the function must be evaluated. The proof thus consists essentially of a comparison of the subobjects of cocycle triples and of cohomology triples.

Suppose first that $\left(\bar{\partial}_{1}, \bar{\partial}_{2}, \Phi\right) \in \mathcal{H}^{(p)}$ is $\left(a_{1}, a_{2}, \tau_{1}, \tau_{2}\right)$-stable. Let $\left(\bar{\partial}_{1}, \bar{\partial}_{2}, \phi\right)$ be any cocycle triple in $\pi^{-1}\left(\bar{\partial}_{1}, \bar{\partial}_{2}, \Phi\right)$, and let $\left(\bar{\partial}_{1}^{\prime}, \bar{\partial}_{2}^{\prime}, \phi^{\prime}\right)$ be a cocycle subtriple. Then $\phi^{\prime}$ defines a cohomology class, $\Phi^{\prime}$, in $H^{1}\left(X, \operatorname{Hom}\left(\mathcal{E}_{2}^{\prime}, \mathcal{E}_{1}^{\prime}\right)\right)$, and $\left(\bar{\partial}_{1}^{\prime}, \bar{\partial}_{2}^{\prime}, \Phi^{\prime}\right)$ is clearly a cohomology subtriple of $\left(\bar{\partial}_{1}, \bar{\partial}_{2}, \Phi\right)$. Thus, by the stability of $\left(\bar{\partial}_{1}, \bar{\partial}_{2}, \Phi\right)$,

$$
\theta_{a_{1}, a_{2}, \tau_{1}, \tau_{2}}\left(\mathcal{E}_{1}^{\prime}, \mathcal{E}_{2}^{\prime}\right)<0
$$

i.e. $\left(\bar{\partial}_{1}, \bar{\partial}_{2}, \phi\right)$ is $\left(a_{1}, a_{2}, \tau_{1}, \tau_{2}\right)$-stable.

Conversely, suppose that all cocycle triples, $\left(\bar{\partial}_{1}, \bar{\partial}_{2}, \phi\right)$, in $\pi^{-1}\left(\bar{\partial}_{1}, \bar{\partial}_{2}, \Phi\right)$ are $\left(a_{1}, a_{2}, \tau_{1}, \tau_{2}\right)$-stable. Let $\left(\bar{\partial}_{1}^{\prime}, \bar{\partial}_{2}^{\prime}, \Phi^{\prime}\right)$ be any cohomology subtriple. Fix any representatives $\phi^{\prime}$ (resp. $\left.\phi\right)$ of $\Phi^{\prime}(\operatorname{resp} . \Phi)$. Then the condition $i_{1 *}\left(\Phi^{\prime}\right)=$ 
$i_{2}^{*}(\Phi)$ (cf. Definition 2.5) implies that $i_{1} \circ \phi^{\prime}=\phi \circ i_{2}+\left(\bar{\partial}_{1} \circ \alpha^{\prime}-\alpha^{\prime} \circ \bar{\partial}_{2}^{\prime}\right)$, for some $\alpha^{\prime} \in \Omega^{0}\left(X, \operatorname{Hom}\left(E_{2}^{\prime}, E_{1}\right)\right)$. Let $\alpha \in \Omega^{0}\left(X, \operatorname{Hom}\left(E_{2}, E_{1}\right)\right)$ be any element such that $\alpha \circ i_{2}=\alpha^{\prime}$. Then, since $i_{2}$ is a holomorphic map, we get

$$
\begin{aligned}
\bar{\partial}_{1} \circ \alpha^{\prime}-\alpha^{\prime} \circ \bar{\partial}_{2}^{\prime} & =\bar{\partial}_{1} \circ \alpha \circ i_{2}-\alpha \circ i_{2} \circ \bar{\partial}_{2}^{\prime} \\
& =\bar{\partial}_{1} \circ \alpha \circ i_{2}-\alpha \circ \bar{\partial}_{2} \circ i_{2} \\
& =\bar{\partial}_{1,2}(\alpha) \circ i_{2} .
\end{aligned}
$$

That is, $i_{1} \circ \phi^{\prime}=\left(\phi+\bar{\partial}_{1,2}(\alpha)\right) \circ i_{2}$, and hence $\left(\bar{\partial}_{1}^{\prime}, \bar{\partial}_{2}^{\prime}, \phi^{\prime}\right)$ is a cocycle subtriple of $\left(\bar{\partial}_{1}, \bar{\partial}_{2}, \phi+\bar{\partial}_{1,2}(\alpha)\right)$. Since $\pi\left(\bar{\partial}_{1}, \bar{\partial}_{2}, \phi+\bar{\partial}_{1,2}(\alpha)\right)=\left(\bar{\partial}_{1}, \bar{\partial}_{2}, \Phi\right)$, it now follows from the stability of this cocycle triple that $\theta_{a_{1}, a_{2}, \tau_{1}, \tau_{2}}\left(\mathcal{E}_{1}^{\prime}, \mathcal{E}_{2}^{\prime}\right)<0$, i.e. $\left(\bar{\partial}_{1}, \bar{\partial}_{2}, \Phi\right)$ is $\left(a_{1}, a_{2}, \tau_{1}, \tau_{2}\right)$-stable.

\subsection{Metric equations.}

In this section we describe the metric equations corresponding to the above definitions of stability. Recall that for a triple $\left(\mathcal{E}_{1}, \mathcal{E}_{2}, \Phi\right)$ with $\Phi \in$ $H^{0}\left(X, \operatorname{Hom}\left(E_{2}, E_{1}\right)\right)$, there is a Hitchin-Kobayashi correspondence between stability (as defined in [BGP]) and metrics satisfying the coupled vortex equations. As equations for metrics $H_{1}$ and $H_{2}$ on $E_{1}$ and $E_{2}$, these are

$$
\begin{aligned}
& i \Lambda F_{H_{1}}+\Phi \Phi^{*}=\tau_{1} \mathbf{I}_{1}, \\
& i \Lambda F_{H_{2}}-\Phi^{*} \Phi=\tau_{2} \mathbf{I}_{2},
\end{aligned}
$$

where $\Phi^{*}$ denotes the adjoint with respect to the metrics $H_{1}$ and $H_{2}$. To obtain the analogous equations corresponding to $\left(a_{1}, a_{2}, \tau_{1}, \tau_{2}\right)$-stability of a $p$-cohomology triple, we need the following operations on form-valued sections of bundles over Kähler manifolds (cf. [W]).

$$
\begin{gathered}
\wedge: \Omega^{p, q}(X, E) \times \Omega^{k, l}\left(X, E^{*}\right) \longrightarrow \Omega^{p+k, q+l}(X, \mathbb{C}) \\
\circ: \Omega^{p, q}\left(X, \operatorname{Hom}\left(E_{1}, E_{2}\right)\right) \times \Omega^{k, l}\left(X, \operatorname{Hom}\left(E_{2}, E_{1}\right)\right) \\
\longrightarrow \Omega^{p+k, q+l}\left(X, \operatorname{Hom}\left(E_{1}, E_{1}\right)\right) \\
\bar{*}_{E}: \Omega^{p, q}(X, E) \longrightarrow \Omega^{n-p, n-q}\left(X, E^{*}\right)
\end{gathered}
$$


These are defined such that for $\phi_{i} \in \Omega^{p, q}(X, E)$,

$$
\phi_{1} \wedge \bar{*}_{E} \phi_{2}=\left(\phi_{1}, \phi_{2}\right) \frac{\omega^{n}}{n !}
$$

where $\omega$ is the Kähler form, and $\left(\phi_{1}, \phi_{2}\right)$ is the inner product coming from the metric on $E$ and the metric on forms of type $(p, q)$. Also, for $\phi_{i} \in$ $\Omega^{p, q}\left(X, \operatorname{Hom}\left(E_{1}, E_{2}\right)\right)$, we have

$$
\phi_{1} \wedge \bar{*}_{E} \phi_{2}=\operatorname{Tr}\left(\phi_{1} \circ \bar{*}_{E} \phi_{2}\right)
$$

Definition 2.11. Given a $p$-cohomology triple $\left(\mathcal{E}_{1}, \mathcal{E}_{2}, \Phi\right)$, and real parameters $\left(a_{1}, a_{2}, \tau_{1}, \tau_{2}\right)$ we define the following equations for metrics on $E_{1}$ and $E_{2}$ and a representative $\phi \in \Omega^{0, p}\left(X, \operatorname{Hom}\left(E_{2}, E_{1}\right)\right)$ of the cohomology class $\Phi$ :

$$
\begin{gathered}
i \Lambda a_{1} F_{H_{1}}+\Lambda^{n}\left(\phi \circ \bar{*}_{E} \phi\right)=\tau_{1} \mathbf{I}_{1}, \\
i \Lambda a_{2} F_{H_{2}}-(-1)^{p} \Lambda^{n}\left(\bar{*}_{E} \phi \circ \phi\right)=\tau_{2} \mathbf{I}_{2}, \\
\bar{\partial}_{1,2}^{*}(\phi)=0 .
\end{gathered}
$$

Remarks 2.12.

2.12.1 The sign of the terms involving $\phi$ are chosen such that $\operatorname{Tr}\left(\Lambda^{n}\left(\phi \circ \bar{*}_{E} \phi\right)\right)$ and $\operatorname{Tr}\left((-1)^{p} \Lambda^{n}\left(\bar{*}_{E} \phi \circ \phi\right)\right)$ are positive. This will be important in section 2.6 .

2.12.2 The coefficients $a_{1}$ and $a_{2}$ will be assumed non-negative, and the parameters $\left(a_{1}, a_{2}, \tau_{1}, \tau_{2}\right)$ must satisfy the constraint

$$
a_{1} d_{1}+a_{2} d_{2}-\tau_{1} r_{1}-\tau_{2} r_{2}=0 .
$$

2.12.3 The coupled vortex equations given in [BGP] correspond to the case $p=0$ and $a_{1}=a_{2}=1$. There is however no good reason to single out these special values for $a_{1}, a_{2}$. This is most clearly seen in the symplectic interpretation of the equations, and will be discussed in the next section. We remark in passing that there is no need to add scale factors to the terms involving $\phi$ since these can be absorbed in $\phi$, or by a rescaling of the metrics. 


\subsection{Moment maps.}

If we fix metrics $K_{1}$ and $K_{2}$ on $E_{1}$ and $E_{2}$, we can reduce the gauge groups to the real unitary groups $\mathfrak{G}_{1}$ and $\mathfrak{G}_{2}$. In addition, $\mathcal{C}_{i}, i=1,2$ and $\Omega^{0, p}\left(X, \operatorname{Hom}\left(E_{2}, E_{1}\right)\right)$ acquire symplectic structures in the usual way. We denote these by $\omega_{1}, \omega_{2}$, and $\omega_{(0, p)}$ respectively. A symplectic form on

$$
\chi^{(p)}=\mathcal{C}_{1} \times \mathcal{C}_{2} \times \Omega^{0, p}\left(\operatorname{Hom}\left(E_{2}, E_{1}\right)\right)
$$

can be produced by taking the sum $\omega_{1}+\omega_{2}+\omega_{(0, p)}$. This is, however, merely one possibility; given any real positive numbers $a_{1}$ and $a_{2}$, we can form a symplectic structures on $\chi^{(p)}$ by defining

$$
\omega_{a_{1}, a_{2}}=a_{1} \omega_{1}+a_{2} \omega_{2}+\omega_{(0, p)} .
$$

Lemma 2.13. The group $\mathfrak{G}_{1} \times \mathfrak{G}_{2}$ acts symplectically on $\left(\chi, \omega_{a_{1}, a_{2}}\right)$, and has a moment map

$$
\Psi_{a_{1}, a_{2}}: \chi \longrightarrow \mathfrak{g}_{1} \times \mathfrak{g}_{2}
$$

given by

$\Psi_{a_{1}, a_{2}}\left(\bar{\partial}_{1}, \bar{\partial}_{2}, \phi\right)=\left(a_{1} \Lambda F_{K_{1}}-i \Lambda^{n}\left(\phi \circ \bar{*}_{E} \phi\right), a_{2} \Lambda F_{K_{2}}-i(-1)^{p} \Lambda^{n}\left(\bar{\aleph}_{E} \phi \circ \phi\right)\right.$.

Proof. Exactly the same as for the $p=0$ case. The sign factor $(-1)^{p}$ comes from interchanging the order in a wedge product forms of type $(0, p)$ and $(n, n-p)$.

With $\mathcal{Z}^{(p)}$ as in Definition 2.1, define $\mathcal{H}_{a_{1}, a_{2}, \tau_{1}, \tau_{2}}^{e q} \subset \mathcal{Z}^{(p)}$ to be the subset on which solutions to equations $(2.4 .7 \mathrm{a}, \mathrm{b})$ can be found. Also define $\mathcal{H a r}{ }^{(p)} \subset \mathcal{Z}^{(p)}$ to be the subset on which solutions to equation $(2.4 .7 \mathrm{c})$ can be found. Then we have

Proposition 2.14. There is a bijective correspondence

$$
\mathcal{H}_{a_{1}, a_{2}, \tau_{1}, \tau_{2}}^{e q} / \mathfrak{G}_{\mathbb{C}}^{(1)} \times \mathfrak{G}_{\mathbb{C}}^{(2)} \longleftrightarrow\left(\Psi_{a_{1}, a_{2}}^{-1}\left(-i \tau_{1},-i \tau_{2}\right) \cap \mathcal{H a r}{ }^{(p)}\right) / \mathfrak{G}_{1} \times \mathfrak{G}_{2} .
$$

Unfortunately, there does not seem to be a way to realize the harmonicity condition as a moment map condition.

\subsection{Hitchin-Kobayashi correspondence.}

In this section we show how our stability conditions follow as a consequence from the existence of solutions to the appropriate metric equations. 
Lemma 2.15. Let $\left(\mathcal{E}_{1}, \mathcal{E}_{2}, \phi\right)$ be a p-cocycle triple (so $\phi \in \Omega^{0, p}\left(\operatorname{Hom}\left(\mathcal{E}_{2}, \mathcal{E}_{1}\right)\right)$ and $\bar{\partial}(\phi)=0)$. Let $\left(a_{1}, a_{2}, \tau_{1}, \tau_{2}\right)$ be any set of real numbers with $a_{i} \geq 0$ for $i=1,2$, and such that

$$
a_{1} d_{1}+a_{2} d_{2}-\tau_{1} r_{1}-\tau_{2} r_{2}=0 .
$$

Suppose there are bundle metrics $H_{1}$ and $H_{2}$ which satisfy the coupled equations (2.4.7a,b), i.e.

$$
\begin{gathered}
i \Lambda a_{1} F_{H_{1}}+\Lambda^{n}\left(\phi \circ \bar{*}_{E} \phi\right)=\tau_{1} \mathbf{I}_{1}, \\
i \Lambda a_{2} F_{H_{2}}-(-1)^{p} \Lambda^{n}\left(\bar{*}_{E} \phi \circ \phi\right)=\tau_{2} \mathbf{I}_{2} .
\end{gathered}
$$

Let $\left(\mathcal{E}_{1}^{\prime}, \mathcal{E}_{2}^{\prime}, \phi^{\prime}\right)$ be a locally free subtriple, i.e. suppose that $i_{1}: \mathcal{E}_{1}^{\prime} \hookrightarrow \mathcal{E}_{1}$ is a subbundle of $\mathcal{E}_{1}, i_{2}: \mathcal{E}_{2}^{\prime} \hookrightarrow \mathcal{E}_{2}$ is a subbundle of $\mathcal{E}_{2}$, and $\phi^{\prime} \in$ $\Omega^{0,1}\left(\operatorname{Hom}\left(\mathcal{E}_{2}^{\prime}, \mathcal{E}_{1}^{\prime}\right)\right)$ satisfies the condition $i_{1} \circ \phi^{\prime}=\phi \circ i_{2}$.

Then

$$
\theta_{a_{1}, a_{2}, \tau_{1}, \tau_{2}}\left(\mathcal{E}_{1}^{\prime}, \mathcal{E}_{2}^{\prime}\right) \leq 0
$$

with equality if and only if $\left(\mathcal{E}_{1}, \mathcal{E}_{2}, \phi\right)$ splits with $\left(\mathcal{E}_{1}^{\prime}, \mathcal{E}_{2}^{\prime}, \phi^{\prime}\right)$ as a direct summand.

Proof. Using the metrics $H_{1}$ and $H_{2}$ we can make orthogonal decompositions $\mathcal{E}_{1}=\mathcal{E}_{1}^{\prime} \oplus\left(\mathcal{E}_{1} / \mathcal{E}_{1}^{\prime}\right)$ and $\mathcal{E}_{2}=\mathcal{E}_{2}^{\prime} \oplus\left(\mathcal{E}_{2} / \mathcal{E}_{2}^{\prime}\right)$. With respect to these decompositions we can write $\phi$ as

$$
\phi=\left(\begin{array}{cc}
\phi^{\prime \prime} & \phi^{\perp} \\
\phi^{\perp^{\prime}} & \phi^{\perp \perp}
\end{array}\right)
$$

In view of the condition $i_{1} \circ \phi^{\prime}=\phi \circ i_{2}$, it follows that in (2.6.1) we have $\phi^{\prime \prime}=\phi^{\prime}$ and $\phi^{\perp^{\prime}}=0$, i.e.

$$
\phi=\left(\begin{array}{cc}
\phi^{\prime} & \phi^{\prime \perp} \\
0 & \phi^{\perp \perp}
\end{array}\right)
$$

The conclusion now follows precisely as in the case of ordinary triples. More specifically, after writing the curvature terms with respect to the above orthogonal decompositions of the bundles, the equations $(2.4 .7 \mathrm{a}, \mathrm{b})$ yield the following:

$$
\begin{gathered}
i a_{1} \Lambda F_{H_{1}}^{\prime}+i a_{1} \Lambda \Pi_{1}+\Lambda^{n}\left(\phi^{\prime \prime} \circ \bar{*}_{E} \phi^{\prime \prime}\right)+\Lambda^{n}\left(\phi^{\prime} \wedge^{{ }^{*}} E \phi^{\prime}\right)=\tau_{1} \mathbf{I}_{1}^{\prime} \\
i a_{2} \Lambda F_{H_{2}}^{\prime}+i a_{2} \Lambda \Pi_{2}-(-1)^{p} \Lambda^{n}\left(\phi^{\prime \prime} \wedge \bar{*}_{E} \phi^{\prime \prime}\right)=\tau_{2} \mathbf{I}_{2}^{\prime}
\end{gathered}
$$


We can take the trace of these equations, and use the fact that for any section $\psi \in \Omega^{0, p}\left(\operatorname{Hom}\left(\mathcal{E}_{2}, \mathcal{E}_{1}\right)\right)$ we have

$$
\operatorname{Tr}\left((-1)^{p} \Lambda^{n} \bar{*}_{E} \psi \wedge \psi\right)=\operatorname{Tr}\left(\Lambda^{n} \psi \wedge \bar{*}_{E} \psi\right)=|\psi|^{2} .
$$

This gives

$$
a_{1} d_{1}^{\prime}+a_{2} d_{2}^{\prime}+a_{1} \operatorname{Tr}\left(i \Lambda \Pi_{1}\right)+a_{2} \operatorname{Tr}\left(i \Lambda \Pi_{2}\right)+\left|\phi^{\prime}\right|^{2}=\tau_{1} r_{1}^{\prime}+\tau_{2} r_{2}^{\prime}
$$

The conclusion follows directly from this, since both $\operatorname{Tr}\left(i \Lambda \Pi_{1}\right)$ and $\operatorname{Tr}\left(i \Lambda \Pi_{2}\right)$ are non-negative.

We now consider $p$-cohomology triples over Riemann surfaces.

Theorem 2.16. Let $\left(\mathcal{E}_{1}, \mathcal{E}_{2}, \Phi\right)$ be a p-cohomology triple over a Riemann surface $X$ (so $\Phi \in H^{p}\left(\operatorname{Hom}\left(\mathcal{E}_{2}, \mathcal{E}_{1}\right)\right)$ ). Let $\left(a_{1}, a_{2}, \tau_{1}, \tau_{2}\right)$ be any set of real numbers with $a_{i} \geq 0$ and satisfying the constraint

$$
a_{1} d_{1}+a_{2} d_{2}-\tau_{1} r_{1}-\tau_{2} r_{2}=0 .
$$

Suppose there is a representative $\phi \in \Omega^{0, p}\left(\operatorname{Hom}\left(\mathcal{E}_{2}, \mathcal{E}_{1}\right)\right)$ for $\Phi$, and bundle metrics $H_{1}$ and $H_{2}$, which satisfy the coupled equations (2.4.7a-c). Then $\left(\mathcal{E}_{1}, \mathcal{E}_{2}, \Phi\right)$ is $\left(a_{1}, a_{2}, \tau_{1}, \tau_{2}\right)$-stable.

Proof. Since $X$ is a Riemann surface, all saturated subtriples are locally free. Let $\left(\mathcal{E}_{1}^{\prime}, \mathcal{E}_{2}^{\prime}, \Phi^{\prime}\right)$ be any such subtriple. To prove the Theorem we need to show that

$$
\theta_{a_{1}, a_{2}, \tau_{1}, \tau_{2}}\left(\mathcal{E}_{1}^{\prime}, \mathcal{E}_{2}^{\prime}\right)<0 .
$$

Notice that by equation (2.4.7c), $\phi$ is the harmonic representative of $\Phi$ with respect to the metrics $H_{1}$ and $H_{2}$. Using the induced metrics on $\mathcal{E}_{1}^{\prime}$ and $\mathcal{E}_{2}^{\prime}$, take the harmonic representative, $\phi^{\prime}$, of $\Phi^{\prime}$. We claim that $\left(\mathcal{E}_{1}^{\prime}, \mathcal{E}_{2}^{\prime}, \phi^{\prime}\right)$ is a subtriple of the 1-cocycle triple $\left(\mathcal{E}_{1}, \mathcal{E}_{2}, \phi\right)$, i.e. we claim that $\phi \circ i_{2}=i_{1} \circ \phi^{\prime}$ where $i_{1}: \mathcal{E}_{1}^{\prime} \hookrightarrow \mathcal{E}_{1}$ and $i_{2}: \mathcal{E}_{2}^{\prime} \hookrightarrow \mathcal{E}_{2}$ are the inclusions. This will prove the proposition, since then by Lemma 2.12, we have $\theta_{a_{1}, a_{2}, \tau_{1}, \tau_{2}}\left(\mathcal{E}_{1}^{\prime}, \mathcal{E}_{2}^{\prime}\right)<0$.

We now prove our claim. From the very definition of the maps induced by $i_{1}$ and $i_{2}$ in cohomology, we get that $\phi \circ i_{2}=i_{1} \circ \phi^{\prime}+\bar{\partial}(\alpha)$, where $\alpha \in \Omega^{0}\left(\operatorname{Hom}\left(\mathcal{E}_{2}^{\prime}, \mathcal{E}_{1}\right)\right)$ and $\bar{\partial}$ denotes the operator induced by $\bar{\partial}_{2}^{\prime}$ and $\bar{\partial}_{1}$. With respect to the orthogonal decompositions $\mathcal{E}_{1}=\mathcal{E}_{1}^{\prime} \oplus\left(\mathcal{E}_{1} / \mathcal{E}_{1}^{\prime}\right)$ and $\mathcal{E}_{2}=$ $\mathcal{E}_{2}^{\prime} \oplus\left(\mathcal{E}_{2} / \mathcal{E}_{2}^{\prime}\right)$ we can thus write 


$$
\phi=\left(\begin{array}{cc}
\phi^{\prime}+\beta^{\prime \prime} & \phi^{\prime} \\
\beta^{\perp^{\prime}} & \phi^{\perp \perp}
\end{array}\right)
$$

where $\bar{\partial}(\alpha)=\beta^{\prime \prime}+\beta^{\perp^{\prime}}$. But harmonic representative are norm minimizing. Thus $\left\|\phi^{\prime}+\beta^{\prime \prime}\right\|^{2} \geq\left\|\phi^{\prime}\right\|^{2}$, and therefore

$$
\|\phi\|^{2} \geq\left\|\begin{array}{cc}
\phi^{\prime} & \phi^{\prime} \perp \\
0 & \phi^{\perp \perp}
\end{array}\right\|^{2}
$$

Thus we get $\|\phi\|^{2} \geq\|\phi-\bar{\partial}(\alpha)\|^{2}$, which is a contradiction unless $\bar{\partial}(\alpha)=0$.

\section{Extensions.}

The case of 1-cohomology triples deserves special attention because of the fact that a 1-cohomology class $\Phi \in H^{1}\left(\operatorname{Hom}\left(\mathcal{E}_{2}, \mathcal{E}_{1}\right)\right)$ can be interpreted as an extension class for extensions of $\mathcal{E}_{2}$ by $\mathcal{E}_{1}$. This can be exploited to study moduli space questions for the set of all such extensions, i.e. for the set of all short exact sequences

$$
0 \longrightarrow \mathcal{E}_{1} \longrightarrow \mathcal{E} \longrightarrow \mathcal{E}_{2} \longrightarrow 0
$$

where $\mathcal{E}_{1}$ and $\mathcal{E}_{2}$ have fixed underlying smooth bundles (denoted by $E_{1}$ and $E_{2}$ respectively).

Such extensions can also be considered from the point of view of the bundle $\mathcal{E}$. This leads to a metric problem and definition of stability that appear somewhat different to the ones considered in the previous section. In this section we discuss such an approach. In the next section we indicate the relationship between the two approaches.

Let us begin therefore with a compact Kähler manifold $X$, and a holomorphic bundle $\mathcal{E} \longrightarrow X$ given as an extensions of bundles as in $(e)$.

\subsection{Stability.}

To formulate the stability condition, we consider extensions as objects in the category of short exact sequences of coherent sheaves of the form

$$
0 \longrightarrow \mathcal{F}_{1} \longrightarrow \mathcal{F} \longrightarrow \mathcal{F}_{2} \longrightarrow 0
$$


Definition 3.1. A morphism of two extensions is defined by the commutative diagram

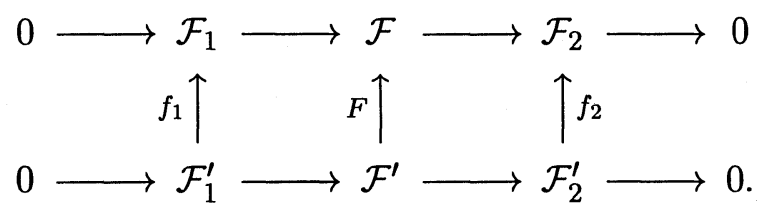

A subobject of $(f)$ consists then of an extension

$$
0 \longrightarrow \mathcal{F}_{1}^{\prime} \longrightarrow \mathcal{F}^{\prime} \longrightarrow \mathcal{F}_{2}^{\prime} \longrightarrow 0
$$

and injective maps $i_{1}, i_{2}, i$ such that the following diagram commutes

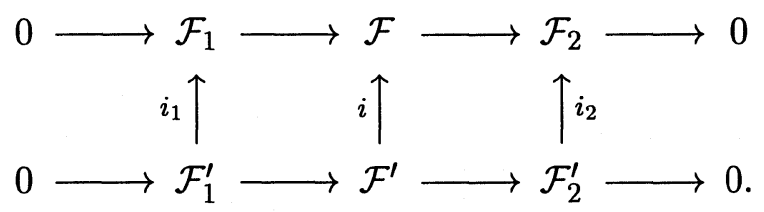

The extension $\left(f^{\prime}\right)$ will be called a subextension of $(f)$.

Lemma 3.2. Let us consider the extension $(f)$. Any subsheaf $i: \mathcal{F}^{\prime} \hookrightarrow \mathcal{F}$ defines a subextension of $(f)$.

Proof. Let $g: \mathcal{F}^{\prime} \longrightarrow \mathcal{F}_{2}$ be the map obtained by composing $i$ with the surjection $\mathcal{F} \longrightarrow \mathcal{F}_{2}$. Then

$$
0 \longrightarrow \operatorname{Ker} g \longrightarrow \mathcal{F}^{\prime} \longrightarrow \operatorname{Im} g \longrightarrow 0
$$

is the desired subextension.

There is hence a one-to-one correspondence between subsheaves of $\mathcal{F}$ and subextensions of $(f)$.

Definition 3.3. Let $e$ be the extension

$$
0 \longrightarrow \mathcal{E}_{1} \longrightarrow \mathcal{E} \longrightarrow \mathcal{E}_{2} \longrightarrow 0
$$

and $e^{\prime}$ the subextension

$$
0 \longrightarrow \mathcal{E}_{1}^{\prime} \longrightarrow \mathcal{E}_{1}^{\prime} \longrightarrow \mathcal{E}_{2}^{\prime} \longrightarrow 0 .
$$

For $\alpha \in \mathbb{R}$ we define the $\alpha$-slope of $e^{\prime}$ as

$$
\mu_{\alpha}\left(e^{\prime}\right)=\mu\left(\mathcal{E}^{\prime}\right)+\alpha \frac{\operatorname{rank} \mathcal{E}_{2}^{\prime}}{\operatorname{rank} \mathcal{E}^{\prime}} .
$$


Definition 3.4. The extension $e$ is said to be $\alpha$-stable (resp. semistable) if and only if for every subextension $e^{\prime} \subset e\left(\right.$ resp. $e^{\prime} \subseteq e$ )

$$
\mu_{\alpha}\left(e^{\prime}\right)<\mu_{\alpha}(e) \quad(\text { resp. } \leq) \text {. }
$$

Remark 3.5. If $\alpha=0$, then $\alpha$-stability is equivalent to ordinary stability.

Proposition 3.6. Let e be $\alpha$-stable, then

$$
\alpha>\mu\left(\mathcal{E}_{1}\right)-\mu\left(\mathcal{E}_{2}\right) .
$$

Conversely, there is some $\epsilon>0$ such that for $\alpha$ in the interval

$$
\left(\mu\left(E_{1}\right)-\mu\left(E_{2}\right), \mu\left(E_{1}\right)-\mu\left(E_{2}\right)+\epsilon\right),
$$

the following is satisfied:

(1) If $e$ is $\alpha$-stable, then $\mathcal{E}_{1}$ and $\mathcal{E}_{2}$ are semistable.

(2) If $\mathcal{E}_{1}$ and $\mathcal{E}_{2}$ are stable then e is $\alpha$-stable.

Proof. For the first statement it suffices to apply the numerical stability condition to the trivial subextension

$$
0 \longrightarrow \mathcal{E}_{1} \longrightarrow \mathcal{E}_{1} \longrightarrow 0 \longrightarrow 0
$$

The proof of the second statement is essentially identical to that of Proposition 2.9. In fact, in view of the results of section 4.2, this result can be treated as a special case of Proposition 2.9, corresponding to the case $p=1, a_{1}=a_{2}=1$. We can also give a direct proof which depends on an examination of the $\alpha$-stability condition for special sub-objects. In this case the subobjects are subextensions with either $\mathcal{E}_{2}^{\prime}=0$ or $\mathcal{E}_{1}^{\prime}=\mathcal{E}_{1}$.

\subsection{Metric equations.}

Given an extension

(e)

$$
0 \longrightarrow \mathcal{E}_{1} \longrightarrow \mathcal{E} \longrightarrow \mathcal{E}_{2} \longrightarrow 0,
$$

the natural metric problem is to look for a metric $H$ on $\mathcal{E}$ satisfying the equation

$$
i \Lambda F_{H}=\left(\begin{array}{cc}
\tau_{1} \mathbf{I}_{1} & 0 \\
0 & \tau_{2} \mathbf{I}_{2}
\end{array}\right)
$$


Here $\tau_{1}$ and $\tau_{2}$ are real numbers an $\mathbf{I}_{1}$ and $\mathbf{I}_{2}$ are the identity endomorphisms in $E_{1}$ and $E_{2}$ respectively. We can make sense of the right hand side since the metric $H$ on $\mathcal{E}$ gives a $C^{\infty}$ splitting of $(e)$, i.e. an identification of the smooth underlying bundle to $\mathcal{E}$ with $E_{1} \oplus E_{2}$.

Remark. If $\tau_{1}=\tau_{2}=\lambda$, equation (3.2.1) reduces to the Hermitian-Einstein equation.

Proposition 3.7. If $H$ satisfies (3.2.1), then the parameters $\tau_{1}$ and $\tau_{2}$ are related by

$$
r_{1} \tau_{1}+r_{2} \tau_{2}=d_{1}+d_{2}
$$

where $r_{1}=\operatorname{rank} \mathcal{E}_{1}, r_{2}=\operatorname{rank} \mathcal{E}_{2}, d_{1}=\operatorname{deg} \mathcal{E}_{1}$ and $d_{2}=\operatorname{deg} \mathcal{E}_{2}$.

Proof. This is easily proved by taking the trace in both sides of (3.2.1) and integrating.

\subsection{Hitchin-Kobayashi correspondence.}

We first prove that $\alpha$-stability is a necessary condition for existence of solutions to the equation (3.2.1).

Proposition 3.8. Let $e$ be the extension of vector bundles

$$
0 \longrightarrow \mathcal{E}_{1} \longrightarrow \mathcal{E} \longrightarrow \mathcal{E}_{2} \longrightarrow 0
$$

and let $\tau_{1}$ and $\tau_{2}$ satisfy (3.2.2). Set $\alpha=\tau_{1}-\tau_{2}$. If $\mathcal{E}$ is indecomposable and admits a metric $H$ satisfying the metric equation for extensions (3.2.1), then e is $\alpha$-stable.

Proof. We need to show that $\mu_{\alpha}\left(e^{\prime}\right)<\mu_{\alpha}(e)$ for every subextension $e^{\prime}$

$$
0 \longrightarrow \mathcal{E}_{1}^{\prime} \longrightarrow \mathcal{E}_{1}^{\prime} \longrightarrow \mathcal{E}_{2}^{\prime} \longrightarrow 0
$$

The proof is a minor modification of the analogous result for the ordinary Hitchin-Kobayashi correspondence. Consider first the locally free subextensions, i.e. the $e^{\prime}$ in which $\mathcal{E}_{1}^{\prime}$ and $\mathcal{E}_{2}^{\prime}$ are locally free. Denote the underlying smooth bundle for $\mathcal{E}^{\prime}$ by $E^{\prime}$, and let $E^{\perp}$ be its orthogonal complement 
with respect to $H$. Then with respect to the smooth orthogonal splitting $E=E^{\prime} \oplus E^{\perp}$, we get the block diagonal decomposition

$$
\sqrt{-1} \Lambda F_{H}=\left(\begin{array}{cc}
\sqrt{-1} \Lambda F^{\prime}+\Pi^{\prime} & * \\
* & \sqrt{-1} \Lambda F^{\perp}-\Pi^{\perp}
\end{array}\right)
$$

where $\Lambda F^{\prime}$ and $\Lambda F^{\perp}$ are the induced metric connections on $\mathcal{E}^{\prime}$ and $\mathcal{E}^{\perp}$ respectively, and $\Pi^{\prime}, \Pi^{\perp}$ are positive definite endomorphisms coming from the second fundamental form for the inclusion of $E^{\prime}$ in $E$. With respect to this splitting of $E$, the endomorphism on the right hand side of the metric equation is no longer diagonal, but has the form

$$
\left(\begin{array}{cc}
T^{\prime} & * \\
* & T^{\perp}
\end{array}\right)=A\left(\begin{array}{cc}
\tau_{1} \mathbf{I}_{1} & 0 \\
0 & \tau_{2} \mathbf{I}_{2}
\end{array}\right) A^{-1}
$$

where the matrix $A$ gives the transformation from the frame $E_{1} \oplus E_{2}$ to $E^{\prime} \oplus E^{\perp}$. If we make the further orthogonal decompositions of $E_{1}$ and $E_{2}$ into components in $E^{\prime}$ and $E^{\perp}$, then

$$
E_{1} \oplus E_{2}=E_{1}^{\prime} \oplus E_{1}^{\perp} \oplus E_{2}^{\prime} \oplus E_{2}^{\perp}
$$

and

$$
E^{\prime} \oplus E^{\perp}=E_{1}^{\prime} \oplus E_{2}^{\prime} \oplus E_{1}^{\perp} \oplus E_{2}^{\perp} .
$$

With respect to these frames, the transformation $A$ is represented by

$$
\left(\begin{array}{cccc}
\mathbf{I}_{1}^{\prime} & 0 & 0 & 0 \\
0 & 0 & \mathbf{I}_{2}^{\prime} & 0 \\
0 & \mathbf{I}_{1}^{\perp} & 0 & 0 \\
0 & 0 & 0 & \mathbf{I}_{2}^{\perp}
\end{array}\right) .
$$

In fact, all we need is the trace of $T^{\prime}$. It follows by a straightforward linear algebra computation that

$$
\operatorname{Tr}\left(T^{\prime}\right)=r_{1}^{\prime} \tau_{1}+r_{2}^{\prime} \tau_{2}
$$

where $r_{1}^{\prime}=\operatorname{rank} \mathcal{E}_{1}^{\prime}$ and $r_{2}^{\prime}=\operatorname{rank} \mathcal{E}_{2}^{\prime}$. We apply this to the condition

$$
\sqrt{-1} \Lambda F^{\prime}+\Pi^{\prime}=T^{\prime}
$$

which can be extracted from the full metric equation. After taking the trace and integrating over $X$, we thus get

$$
\int_{X} \operatorname{Tr}\left(\sqrt{-1} \Lambda F^{\prime}\right)+\int_{X} \operatorname{Tr}\left(\Pi^{\prime}\right)=r_{1}^{\prime} \tau_{1}+r_{2}^{\prime} \tau_{2} .
$$


Using the Chern-Weil formula for $\operatorname{deg}\left(\mathcal{E}^{\prime}\right)$, and the positivity of $\Pi^{\prime}$, we obtain

$$
\operatorname{deg}\left(\mathcal{E}^{\prime}\right) \leq r_{1}^{\prime} \tau_{1}+r_{2}^{\prime} \tau_{2}
$$

with equality if and only if $\Pi^{\prime}=0$, i.e. if and only if $\mathcal{E}$ splits. If $\alpha=\tau_{1}-\tau_{2}$, and $\mu_{\alpha}\left(\mathcal{E}^{\prime}\right)$ is as in Definition 3.3, then (3.3.5) is equivalent to $\mu_{\alpha}\left(e^{\prime}\right)<\mu_{\alpha}(e)$. This proves the result for locally free subobjects. If $e^{\prime}$ is not locally free, then there is a subvariety $\Sigma \subset X$ of codimension at most two, such that $\left.\mathcal{E}^{\prime}\right|_{X-\Sigma}$ is locally free. We can thus apply the above arguments over $X-\Sigma$. This is good enough, because of the size of the codimension of $\Sigma$.

We now prove that $\alpha$-stability is a sufficient condition for existence of special metrics in the sense defined by equations (3.2.1). That is, we prove

Theorem 3.9. Suppose that $\alpha<0$ and

$$
0 \longrightarrow \mathcal{E}_{1} \longrightarrow \mathcal{E} \longrightarrow \mathcal{E}_{2} \longrightarrow 0
$$

is an $\alpha$-stable extension. Let $\tau_{1}$ and $\tau_{2}$ be such that $\alpha=\tau_{1}-\tau_{2}$ and $\operatorname{deg}(E)=$ $r_{1} \tau_{1}+r_{2} \tau_{2}$. Then there is a metric $H$ on $\mathcal{E}$ satisfying the equation (3.2.1), i.e

$$
i \Lambda F_{H}=\left(\begin{array}{cc}
\tau_{1} \mathbf{I}_{1} & 0 \\
0 & \tau_{2} \mathbf{I}_{2}
\end{array}\right) .
$$

The proof is an adaptation of the methods used in [Do1] (also [S] and [UY]) in proving the Hitchin-Kobayashi correspondence for ordinary stable bundles. As shown by Donaldson, the Hermitian-Einstein equation is the equation satisfied by the critical points of a certain functional defined on the space of Hermitian metrics on $\mathcal{E}$. We shall modify this functional to show that our equations appear in the same way.

Just as in the case of the Hermitian-Einstein equation, we can separate out the trace and trace-free parts of the equation. We can fix the determinant of the metric on $E$ to satisfy the trace part,

$$
i \Lambda \operatorname{Tr}\left(F_{H}\right)=r_{1} \tau_{1}+r_{2} \tau_{2} .
$$

The problem then becomes one of finding a new metric with this same determinant, and which satisfies

$$
i \Lambda F_{H}^{0}=\left(\begin{array}{cc}
\frac{r_{2}}{r} \alpha \mathbf{I}_{1} & 0 \\
0 & -\frac{r_{1}}{r} \alpha \mathbf{I}_{2}
\end{array}\right)
$$


where $\alpha=\tau_{1}-\tau_{2}$.

Recall Donaldson's original functional to prove existence of solutions of the Hermitian-Einstein equation: Let $\mathcal{E}$ be a holomorphic vector bundle over a compact Kähler manifold $(X, \omega)$. Donaldson defined a functional $M(-,-)$ on pairs of Hermitian metrics on $\mathcal{E}$ using Bott-Chern secondary classes. Namely

$$
M(H, K)=\int_{X}\left(R_{2}(H, K)-2 \lambda R_{1}(H, K) \omega\right) \wedge \omega^{n-1},
$$

where

$$
\begin{aligned}
R_{1}(H, K) & =\log \operatorname{det}\left(K^{-1} H\right)=\operatorname{Tr}\left(\log K^{-1} H\right) \\
i \bar{\partial} \partial R_{2}(H, K) & =\left(-\operatorname{Tr}\left(F_{H}^{2}\right)\right)-\left(-\operatorname{Tr}\left(F_{K}^{2}\right)\right), \\
\lambda & =\frac{\operatorname{deg} \mathcal{E}}{\operatorname{rank\mathcal {E}}} .
\end{aligned}
$$

Now fix a smooth background metric $K$, with determinant satisfying (3.3.6). Let

$$
S(K)=\left\{s \in \Omega^{0}(X, \text { End } E) \mid s^{*_{K}}=s, \operatorname{Tr}(s)=0\right\} .
$$

Then any other metric with the same determinant as $K$ can be described by $K e^{s}$, with $s \in S(K)$. Fix an integer $p>2 n$, and define

$$
\mathcal{M} e t_{2}^{p}=\left\{H=K e^{s} s \in L_{2}^{p}(S(K))\right\} .
$$

Let $M: \mathcal{M e t}(\mathcal{E}) \longrightarrow \mathbb{R}$ be given by $M(H)=M(K, H)$. The important property of $M$ is that $H$ is a critical point if and only if $H$ satisfies the trace free part of the Hermitian-Einstein equations, i.e.

$$
i \Lambda F_{H}^{0}=0 .
$$

Consider now the extension

$$
0 \longrightarrow \mathcal{E}_{1} \longrightarrow \mathcal{E} \longrightarrow \mathcal{E}_{2} \longrightarrow 0 .
$$

Given a background metric $K$ on $\mathcal{E}$ we can (smoothly) identify $\mathcal{E}_{2}$ with the orthogonal complement of $\mathcal{E}_{1}$ in $\mathcal{E}$, and in this way get metrics $K_{1}$ and $K_{2}$ 
on $\mathcal{E}_{1}$ and $\mathcal{E}_{2}$ respectively. Any other metric $\mathrm{H}$ can similarly be split into $H_{1}$ and $H_{2}$ (by using an $H$-orthogonal splitting of $\mathcal{E}$ ). Denote

$$
M_{D}(H, K)=\int_{X} R_{2}(H, K) \wedge \omega^{n-1} .
$$

Let $\tau_{1}$ and $\tau_{2}$ be real parameters. We shall consider the functional (3.3.13)

$$
M_{\tau_{1}, \tau_{2}}(H, K)=M_{D}(H, K)-2 \int_{X}\left(\tau_{1} R_{1}\left(H_{1}, K_{1}\right)+\tau_{2} R_{1}\left(H_{2}, K_{2}\right)\right) \wedge \omega^{n} .
$$

Remark. If $\tau_{1}=\tau_{2}=\lambda$, then $M_{\tau_{1}, \tau_{2}}(H, K)=M(H, K)$, as can be easily seen from the following simple fact (see [Do1, Prop. 7. p. 10]).

Lemma 3.10. Let $H$ and $K$ be Hermitian metrics on $\mathcal{E}$ and Let $H_{1}, K_{1}$ and $H_{2}, K_{2}$ the corresponding metrics induced on $\mathcal{E}_{1}$ and $\mathcal{E}_{2}$ respectively, then

$$
R_{1}(H, K)=R_{1}\left(H_{1}, K_{1}\right)+R_{1}\left(H_{2}, K_{2}\right) .
$$

Notice that $R_{1}(H, K)=0$ if the metrics have fixed determinant. We can thus simplify our definition to

$$
M_{\tau_{1}, \tau_{2}}(H, K)=M_{D}(H, K)-2\left(\tau_{1}-\tau_{2}\right) \int_{X} R_{1}\left(H_{1}, K_{1}\right) \wedge \omega^{n} .
$$

Let us fix $K$ and define

$$
M_{\tau_{1}, \tau_{2}}(H)=M_{\tau_{1}, \tau_{2}}(H, K) .
$$

Define $m^{0}: \mathcal{M e t}(\mathcal{E}) \longrightarrow \Omega^{0}(X$, End $E)$ by

$$
m^{0}(H)=\Lambda F_{\bar{\partial}_{E}, H}^{0}+\sqrt{-1} T_{H}^{0},
$$

where, with respect to the orthogonal splitting $E=E_{1} \oplus E_{2}$ determined by $H$,

$$
\begin{aligned}
T_{H}^{0} & =\left(\begin{array}{cc}
\tau_{1} \mathbf{I}_{1} & 0 \\
0 & \tau_{2} \mathbf{I}_{2}
\end{array}\right)-\operatorname{Tr}\left(\begin{array}{cc}
\tau_{1} \mathbf{I}_{1} & 0 \\
0 & \tau_{2} \mathbf{I}_{2}
\end{array}\right) \\
& =\left(\begin{array}{cc}
\frac{r_{2}}{r} \alpha \mathbf{I}_{1} & 0 \\
0 & -\frac{r_{1}}{r} \alpha \mathbf{I}_{2}
\end{array}\right) .
\end{aligned}
$$

The crucial properties of $M_{\tau_{1}, \tau_{2}}$ are described in the next proposition. 
Proposition 3.11. (1) Given any three metrics $H, K, J$, we have

$$
M_{\tau_{1}, \tau_{2}}(H, K)+M_{\tau_{1}, \tau_{2}}(K, J)=M_{\tau_{1}, \tau_{2}}(H, J) .
$$

(2) If $H(t)=H e^{t s}$ with $s \in S(H)$, then

$$
\frac{d}{d t} M_{\tau_{1}, \tau_{2}}(H(t))=2 i \int_{X} \operatorname{Tr}\left(s m^{0}(H(t))\right) .
$$

(3) If $s \in S(H)$ is given by $s=\left(\begin{array}{cc}s_{1} & u \\ u^{*} & s_{2}\end{array}\right)$ with respect to the orthogonal splitting $E=E_{1} \oplus E_{2}$ determined by $H$, then

$$
\begin{aligned}
\left.\frac{d^{2}}{d t^{2}} M_{\tau_{1}, \tau_{2}}(H(t))\right|_{t=0} & =2 i \int_{X} \operatorname{Tr}\left(\left.s \frac{d}{d t} m^{0}(H(t))\right|_{t=0}\right) \\
& =\left\|D_{H}^{\prime}(s)\right\|^{2}-\alpha\|u\|^{2}
\end{aligned}
$$

Proof. (1) This follows immediately from the properties of the Bott-Chern classes.

(2) Chose a frame for $E$ such that $H$ can be written as

$$
\left(\begin{array}{cc}
H_{1} & 0 \\
0 & H_{2}
\end{array}\right)
$$

that is a frame in which $\mathcal{E}_{1}$ and $\mathcal{E}_{2}$ are $H$-orthogonal. In terms of this frame we can write

$$
s=\left(\begin{array}{cc}
s_{1} & u \\
u^{*} & s_{2}
\end{array}\right)
$$

where $s_{1} \in S\left(H_{1}\right), s_{2} \in S\left(H_{2}\right)$ and $u \in \operatorname{Hom}\left(E_{2}, E_{1}\right)$. We have to show that

$$
\begin{aligned}
\left.\frac{d}{d t} M_{\tau_{1}, \tau_{2}}(H(t))\right|_{t=0} & =2 i \int_{X} \operatorname{Tr}\left(s i \Lambda F_{H}^{0}-\left(\begin{array}{cc}
\frac{r_{2}}{r} \alpha I_{1} & 0 \\
0 & -\frac{r_{1}}{r} \alpha I_{2}
\end{array}\right)\right) \\
& =2 i \int_{X} \operatorname{Tr}\left(s \Lambda F_{H(t)}^{0}\right)-2 i \alpha \int_{X} \operatorname{Tr}\left(s_{1}\right) .
\end{aligned}
$$

From [Do1] we know that $\frac{d}{d t} M_{D}(H(t))=2 i \int_{X} \operatorname{Tr}\left(s \Lambda F_{H(t)}^{0}\right)$, so it remains to compute $\left.\frac{d}{d t} R_{1}\left(H_{1}(t), H_{1}\right)\right|_{t=0}$. If we write

$$
H(t)=H e^{t s}=H\left(\begin{array}{cc}
h_{1}(t) & * \\
* & *
\end{array}\right),
$$


with respect to the $H$-orthogonal frame, then

$$
R_{1}\left(H_{1}(t), H_{1}\right)=\log \operatorname{det} h_{1}(t)=\log \operatorname{det}\left(1+t s_{1}+\frac{t^{2}}{2}\left(s_{1}^{2}+u u^{*}\right)+O\left(t^{3}\right)\right) .
$$

A straightforward computation yields the result

$$
\left.\frac{d}{d t} R_{1}\left(H_{1}(t), H_{1}\right)\right|_{t=0}=\operatorname{Tr}\left(s_{1}\right) .
$$

(3) It follows from (3.3.17) that

$$
\left.\frac{d^{2}}{d t^{2}} R_{1}\left(H_{1}(t), H_{1}\right)\right|_{t=0}=\operatorname{Tr}\left(u u^{*}\right)=|u|^{2} .
$$

The result now follows from this, plus the fact that

$$
\frac{d}{d t} M_{D}(H(t))=\left\|D_{H}^{\prime}(s)\right\|^{2}
$$

Notice that as a consequence of (1) and (3) in Proposition 3.11 we get

Proposition 3.12. Suppose that $\alpha<0$ and (e) is an $\alpha$-stable extension. Then

1.

$$
\frac{d^{2}}{d t^{2}} M_{\tau_{1}, \tau_{2}}(H(t))>0
$$

2. $\operatorname{Ker}(L)=0$, where $L$ is the operator on $L_{2}^{p}(S(H)$ defined by $L(s)=$ $\left.\frac{d}{d t} m^{0}(H(t))\right|_{t=0}$.

Proof. Both of these statements follow from the fact that if $s$ is as in (3) and $L(s)=0$, then $\bar{\partial}_{1}\left(s_{1}\right)=\bar{\partial}_{2}\left(s_{2}\right)=u=0$. The eiegenspaces of $s$ thus split the extension $(e)$ into a direct sum of extensions. This violates the stability criterion, since the $\alpha$-slope inequality cannot be satisfied by both summands.

The functional $M_{\tau_{1}, \tau_{2}}$ thus has the convexity features we require. Furthermore, 
Lemma 3.13. Suppose that $\alpha<0$ and let $H=K e^{s}$ with $s \in L_{2}^{p}(S(K))$. Let $s=\left(\begin{array}{ll}s_{1} & u \\ u^{*} & s_{2}\end{array}\right)$ be the block decomposition of $s$ with respect to the orthogonal splitting $E=E_{1} \oplus E_{2}$ determined by $K$. Let $\Psi: \mathbb{R} \times \mathbb{R} \longrightarrow \mathbb{R}$ be a smooth function as in [B] (or [S]). Then

$$
\begin{aligned}
M_{\tau_{1}, \tau_{2}}(H) & =\sqrt{-1} \int_{X} \operatorname{Tr}\left(s \Lambda F_{K}\right)+\int_{X}\left(\Psi(s) \bar{\partial}_{E} s, \bar{\partial}_{E} s\right)_{K}-2 \alpha R_{1}\left(H_{1}, K_{1}\right) \\
& \geq \sqrt{-1} \int_{X} \operatorname{Tr}\left(s \Lambda F_{K}\right)+\int_{X}\left(\Psi(s) \bar{\partial}_{E} s, \bar{\partial}_{E} s\right)_{K}-\alpha \int_{X} \operatorname{Tr}\left(s_{1}\right)
\end{aligned}
$$

where the meaning of $\Psi(s)$ is as in [B] or [S].

Proof. The first line follows from the computations in [S] (or [Do1]). The second uses the convexity properties of the function $R_{1}\left(H(t)_{1}, K_{1}\right)$, and the fact that its first derivative at $t=0$ is given by $\int_{X}\left(\operatorname{Tr}\left(s_{1}\right)\right.$.

This is slightly weaker than the analogous result for the original Donaldson functional, but is strong enough for our purposes.

The rest of the proof of Theorem 3.9 is precisely along the lines of the analogous result in $[\mathrm{S}]$. We give here a sketch of the main ideas. Fix a real number $B$ such that $\left\|m^{0}(K)\right\|_{L^{p}}^{p} \leq B$ (where $\left\|m^{0}(K)\right\|_{L^{p}}^{p}=$ $\left.\int_{X}\left|m^{0}(K)\right|_{K}^{p} d v o l\right)$. Define

$$
\mathcal{M e t}_{2}^{p}(B)=\left\{H \in \mathcal{M e t}_{2}^{p} \mid\left\|m^{0}(H)\right\|_{L^{p}}^{p} \leq B\right\} .
$$

We look for minima of $M_{\tau_{1}, \tau_{2}}(H)$ on $\mathcal{M e t}_{2}^{p}(B)$. As in the case of the unmodified Donaldson functional, if the extension $(e)$ is $\alpha$-stable, then there are no extrema on the boundary of this constrained space, and the minima occur at solutions to the metric equation $m^{0}(H)=0$.

To show that minima do occur, we need

Proposition 3.14. Either (e) is $\alpha$-stable or we can find positive constants $C_{1}$ and $C_{2}$ such that

$$
\operatorname{Sup}|s|<C_{1} M_{\tau_{1}, \tau_{2}}\left(K e^{s}\right)+C_{2}
$$

for all $K e^{s} \in \mathcal{M} e t_{2}^{p}(B)$. 
Sketch of Proof. As in the case of the unmodified Donaldson functional, one first shows that for metrics in the constrained set $\mathcal{M e t}_{2}^{p}(B)$, the $C^{0}$ estimate given above is equivalent to a $C^{1}$ estimate of the same type. One then supposes that no such estimate holds. It follows that one may find a sequence $\left\{u_{i}\right\} \subset L_{2}^{P}\left(S(K)\right.$ such that $\left\|u_{i}\right\|_{L^{1}}=1$. This has a weakly convergent subsequence in $L_{1}^{2}\left(S(K)\right.$, with non-trivial limit denoted by $u_{\infty}$. One then shows that the eigenvalues of $u_{\infty}$ are constant almost everywhere. This is done, as in [S], by making use of an estimate of the form:

Proposition 3.15. Let $\mathcal{F}: \mathbb{R} \times \mathbb{R} \longrightarrow \mathbb{R}$ be any smooth positive function which satisfies $\mathcal{F}(x, y) \leq 1 /(x-y)$ whenever $x>y$. Then

$$
\sqrt{-1} \int_{X} \operatorname{Tr}\left(u_{\infty} \Lambda F_{K}\right)+\int_{x}\left(\mathcal{F}\left(u_{\infty}\right) \bar{\partial}_{E} u_{\infty}, \bar{\partial}_{E} u_{\infty}\right)_{K}-\alpha \int_{x} \operatorname{Tr}\left(u_{\infty, 1}\right) \leq 0
$$

where $u_{\infty}=\left(\begin{array}{cc}u_{\infty, 1} & * \\ * & *\end{array}\right)$ with respect to the splitting of $E$ determined by $K$.

Proof. This follows from the analysis in [S], plus the estimate given in Lemma 3.13.

Since $\operatorname{Tr}\left(u_{\infty}\right)=0$, there are at least two distinct eigenvalues. Let $\lambda_{1}<$ $\lambda_{2}, \ldots,<\lambda_{k}$ denote the distinct eigenvalues. Setting $a_{i}=\lambda_{i+1}-\lambda_{i}$, one can thus define projections $\pi_{i} \in L_{1}^{2}(S(K))$ such that

$$
u_{\infty}=\lambda_{r} \mathbf{I}-\sum_{i}^{k-1} a_{i} \pi_{i} .
$$

By an important result of Uhlenbeck and Yau (cf. [UY]), the $\pi_{i}$ define a filtration of $\mathcal{E}$ by reflexive subsheaves

$$
\mathcal{E}_{1} \subset \mathcal{E}_{2} \subset \cdots \subset \mathcal{E}_{k}=\mathcal{E}
$$

Each subsheaf $\mathcal{E}_{j}$ determines a subextension

$$
0 \longrightarrow \mathcal{E}_{1, j} \longrightarrow \mathcal{E}_{j} \longrightarrow \mathcal{E}_{2, j} \longrightarrow 0 \text {. }
$$

Now define the numerical quantity

$$
Q=\lambda_{k}\left(r \mu(\mathcal{E})-r_{1} \tau_{1}-r_{2} \tau_{2}\right)-\sum_{i}^{k_{1}} a_{i}\left(r_{i} \mu\left(\mathcal{E}_{i}\right)-r_{1, i} \tau_{1}-r_{2, i} \tau_{2}\right)
$$


where $\mu\left(\mathcal{E}_{i}\right)$ is the slope of $\mathcal{E}_{j}$, and $r_{a, i}$ is the rank of $\mathcal{E}_{a, i}$.

Using Lemma 3.15 and the fact that $u_{\infty}=\lambda_{r} \mathbf{I}-\sum_{i}^{k-1} a_{i} \pi_{i}$, one shows (by precisely the method in [S]) that $Q \leq 0$. On the other hand, $\tau_{1}$ and $\tau_{2}$ are related by $r \mu(\mathcal{E})-r_{1} \tau_{1}-r_{2} \tau_{2}=0$, and if $(e)$ is $\alpha$-stable, then

$$
r_{i} \mu\left(\mathcal{E}_{i}\right)-r_{1, i} \tau_{1}-r_{2, i} \tau_{2}<0
$$

for all $i=1, \ldots, k-1$. Thus $Q>0$ if $(e)$ is $\alpha$-stable. This proves the proposition.

To complete the proof of the Hitchin-Kobayashi correspondence, it remains to show that the minimum of $M_{\tau_{1}, \tau_{2}}$ is a smooth solution to the equation (3.2.1). This is done exactly as in [Do1] or [S].

\subsection{An example.}

As an example, we can consider the case where $E_{1}=L_{1}$ and $E_{2}=L_{2}$ are line bundles over a Riemann surface. We assume further that $d_{1}<d_{2}$, where $d_{i}$ denotes the degree of $L_{i}$. Let us denote such extensions by

$$
0 \longrightarrow \mathcal{L}_{1} \longrightarrow \mathcal{E} \longrightarrow \mathcal{L}_{2} \longrightarrow 0 \text {. }
$$

Since line bundles are automatically stable, Lemma 3.6(1) gives

Lemma 3.16. Let $L_{1}$ and $L_{2}$ be as above. Then there is some $\epsilon>0$ such that all extensions $(l)$ as above are $\alpha$-stable, for any $\alpha$ in the interval

$$
\left(d_{1}-d_{2}, d_{1}-d_{2}+\epsilon\right) \text {. }
$$

We can give a more detailed analysis. The main reason for this is that the possibilities for sub-extensions are so restricted; they all correspond to rank one (i.e. line-) subbundles of $\mathcal{E}$, and are of one of two types. The only possibilities are

$$
0 \longrightarrow \mathcal{L}_{1} \longrightarrow \mathcal{L}_{1} \longrightarrow 0 \longrightarrow 0
$$

or

$$
0 \longrightarrow 0 \longrightarrow \mathcal{L} \longrightarrow \mathcal{L} \longrightarrow 0 .
$$

Computing the $\alpha$-slopes, we see that

$$
\begin{aligned}
\mu_{\alpha}\left(0, \mathcal{L}_{1}\right) & =d_{1} \\
\mu_{\alpha}(\mathcal{L}, 0) & =d_{\mathcal{L}}+\alpha .
\end{aligned}
$$


From this we see that if $(l)$ is $\alpha$-stable, then

$$
0<d_{1}-d_{2}<\alpha<d_{1}+d_{2}-2 d_{\mathcal{L}}
$$

for all subbundles $\mathcal{L} \neq \mathcal{L}_{1}$. Now define

$$
\operatorname{div}(\mathcal{E})=\operatorname{Max}\left\{d_{\mathcal{L}} \mid d_{\mathcal{L}} \text { is the degree of a line subbundle of } \mathcal{E}\right\}
$$

Lemma 3.17. If

$$
0<d_{1}-d_{2}<d_{1}+d_{2}-2 \operatorname{div}(\mathcal{E})
$$

then $(l)$ is $\alpha$-stable for any $\alpha$ in the interval $\left(d_{1}-d_{2}, d_{1}+d_{2}-2 \operatorname{div}(\mathcal{E})\right)$.

Proof. Given any $\alpha$ such that $0<d_{1}-d_{2}<\alpha<d_{1}+d_{2}-2 \operatorname{div}(\mathcal{E})$, we get

$$
d_{1}<\mu_{\alpha}(\mathcal{E})=\left(d_{1}+d_{2}\right) / 2+\alpha / 2
$$

and

$$
d_{\mathcal{L}}+\alpha<\left(d_{1}+d_{2}\right) / 2+\alpha / 2 .
$$

By equations (3.4.3) and (3.4.4), and the above remarks concerning the possible subextensions of $(l)$, this is all we need to check.

Furthermore, the range for $\alpha$ is clearly partitioned into intervals of length 2 , with the boundaries at the values $\left\{d_{1}-d_{2}, d_{1}-d_{2}+2, \ldots, d_{2}-d_{1}-2, d_{2}-\right.$ $\left.d_{1}\right\}$.

Proposition 3.18. Let $L_{1}$ and $L_{2}$ be as above, and let $(l)$ denote an extension as above.

1. For $\alpha$ in the interval $\left(d_{1}-d_{2}, d_{1}-d_{2}+2\right)$, all non-trivial extensions (l) are $\alpha$-stable.

2. Suppose that $\alpha_{1}>\alpha_{2}>d_{1}-d_{2}$. If $(l)$ is $\alpha_{1}$-stable, then it is $\alpha_{2}$-stable.

3. For $\alpha \geq-2$, if $(l)$ is an $\alpha$-stable extension, then $\mathcal{E}$ is a semistable bundle.

4. For $\alpha \geq 0$, if $(l)$ is an $\alpha$-stable extension, then $\mathcal{E}$ is a stable bundle.

5. If $\mathcal{E}$ is a stable (resp. semistable) bundle, then for any $d_{1}-d_{2}<\alpha \leq 0$ (resp. $\left.d_{1}-d_{2}<\alpha<0\right),(l)$ is an $\alpha$-stable extension. 
Proof. Part (1) follows from the fact that $\operatorname{div}(\mathcal{E}) \leq d_{2}$, with equality possible if and only if the extension is the trivial one (cf. [G]). Thus for any nontrivial extension, we have $d_{1}+d_{2}-2 \operatorname{div}(\mathcal{E})>d_{1}-d_{2}$. Now use Lemma 3.16. Part (2) follows from the observation that for any subextension of the type in (3.4.2), we have $\mu_{\alpha}(\mathcal{L}, 0)-\mu_{\alpha}(\mathcal{E})=d_{\mathcal{L}}-\frac{d_{1}+d_{2}}{2}+\frac{\alpha}{2}$. Parts (3) and (4) both follows from the observation that if $(l)$ is $\alpha$-stable, then $\operatorname{div}(\mathcal{E})<\left(d_{1}+d_{2}\right) / 2-\alpha / 2$. Part (5) follows from Lemma 3.16 and the fact that if $\mathcal{E}$ is stable (resp. semistable), then $\operatorname{div}(\mathcal{E})<\left(d_{1}+d_{2}\right) / 2$ (resp. $\operatorname{div}(\mathcal{E}) \leq\left(d_{1}+d_{2}\right) / 2$.

We thus get the following picture. Let

$$
\mathcal{E} x t\left(L_{1}, L_{2}\right)=\left\{\text { all extensions } 0 \longrightarrow \mathcal{L}_{1} \longrightarrow \mathcal{E} \longrightarrow \mathcal{L}_{2} \longrightarrow 0\right\},
$$

and let $\mathcal{E} x t^{*}\left(L_{1}, L_{2}\right) \subset \mathcal{E} x t\left(L_{1}, L_{2}\right)$ denote the non-trivial extensions. Given an integer $k$, define

$$
\mathcal{E} x t_{k}\left(L_{1}, L_{2}\right)=\left\{(l) \in \mathcal{E} x t\left(L_{1}, L_{2}\right) \mid(l) \text { is } \alpha \text {-stable, and } k<\alpha<k+2\right\} .
$$

Set

$$
\mathcal{E} x t_{-}\left(L_{1}, L_{2}\right)= \begin{cases}\mathcal{E} x t_{-2}\left(L_{1}, L_{2}\right) & \text { if }\left(d_{1}-d_{2}\right) \text { is even } \\ \mathcal{E} x t_{-1}\left(L_{1}, L_{2}\right) & \text { if }\left(d_{1}-d_{2}\right) \text { is odd }\end{cases}
$$

and

$$
\mathcal{E} x t_{+}\left(L_{1}, L_{2}\right)=\left\{\begin{array}{ll}
\mathcal{E} x t_{0}\left(L_{1}, L_{2}\right) & \text { if }\left(d_{1}-d_{2}\right) \text { is even } \\
\mathcal{E} x t_{1}\left(L_{1}, L_{2}\right) & \text { if }\left(d_{1}-d_{2}\right) \text { is odd }
\end{array} .\right.
$$

Also define

$$
\mathcal{E} x t_{s}\left(L_{1}, L_{2}\right)=\left\{(l) \in \mathcal{E} x t^{*}\left(L_{1}, L_{2}\right) \mid \mathcal{E} \text { is a stable bundle }\right\}
$$

$$
\mathcal{E}_{x} t_{s s}\left(L_{1}, L_{2}\right)=\left\{(l) \in \mathcal{E} x t^{*}\left(L_{1}, L_{2}\right) \mid \mathcal{E} \text { is a semistable bundle }\right\}
$$

Then we can summarize proposition 3.18 by the diagram

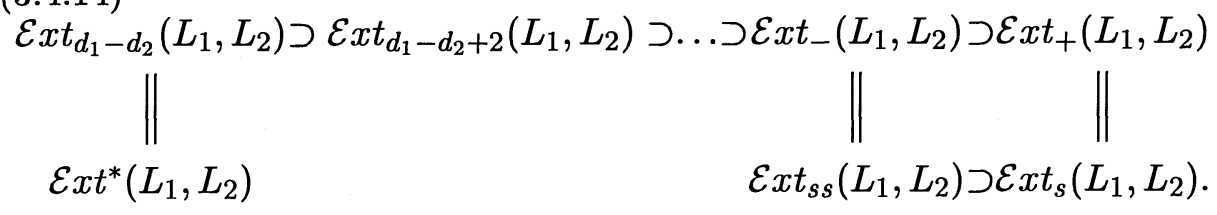




\section{1-Cohomology triples, extensions and surjective triples.}

The correspondence with 1-cohomology triples is not the only way that extensions as in Section 3 are related to triples. Given an extension $(e)$ as in $\S 3$, one can extract a (0-cohomology) triple $\left(\mathcal{E}_{2}, \mathcal{E}, \pi\right)$. Conversely, given a triple $\left(\mathcal{E}_{2}, \mathcal{E}, \pi\right)$ in which $E=E_{1} \oplus E_{2}$ and $\pi$ is surjective, we get an extension of $\mathcal{E}_{2}$ by $\mathcal{E}_{1}=\operatorname{Ker}(\pi)$.

In this section we compare and relate notions of stability, moduli spaces, equations for special metric, etc. for

1. 1-cohomology triples on $\left(E_{1}, E_{2}\right)$,

2. extensions on $\left(E_{1}, E_{2}\right)$, and

3. surjective (0-cohomology) triples on $\left(E_{2}, E\right)$.

\subsection{Configuration spaces.}

We begin with some definitions. Let $E_{1}$ and $E_{2}$ be (as usual) smooth bundles over $X$, and fix $E=E_{1} \oplus E_{2}$ as a smooth bundle. Holomorphic bundles with these as their underlying smooth bundles will be denoted by $\mathcal{E}_{1}, \mathcal{E}_{2}, \mathcal{E}$ respectively.

Definition 4.1. 1. A surjective triple on $\left(E_{2}, E\right)$ is a (0-cohomology) triple, $\left(\mathcal{E}_{2}, \mathcal{E}, \pi\right)$, in which $\pi: \mathcal{E} \longrightarrow \mathcal{E}_{2}$ is a surjective map. Set

$$
\mathcal{H}_{s}\left(E_{2}, E\right)=\left\{\left(\mathcal{E}_{2}, \mathcal{E}, \pi\right): \pi \text { is surjective }\right\} .
$$

2. Denote by $\mathcal{E} x t\left(E_{1}, E_{2}\right)$ the set of all holomorphic structures on $E$ which can be described as extensions of $\mathcal{E}_{2}$ by $\mathcal{E}_{1}$, i.e.

$$
\mathcal{E} x t\left(E_{1}, E_{2}\right)=\left\{0 \longrightarrow \mathcal{E}_{1} \longrightarrow \mathcal{E} \longrightarrow \mathcal{E}_{2} \longrightarrow 0\right\}
$$

Recall also, from $\S 2$, that

$$
\mathcal{H}^{(1)}\left(E_{1}, E_{2}\right)=\left\{\left(\mathcal{E}_{1}, \mathcal{E}_{2}, \Phi\right) \mid \Phi \in H^{1}\left(\operatorname{Hom}\left(\mathcal{E}_{2}, \mathcal{E}_{1}\right)\right)\right\}
$$

is the space of 1-cohomology pairs on $\left(E_{1}, E_{2}\right)$.

On each of $\mathcal{H}_{s}\left(E_{2}, E\right), \mathcal{E} x t\left(E_{1}, E_{2}\right)$, and $\mathcal{H}^{(1)}\left(E_{1}, E_{2}\right)$ there are natural equivalence relations. 
Definition/Lemma 4.2. 1. In $\mathcal{H}^{(1)}\left(E_{1}, E_{2}\right)$, the equivalence relation is given by the action of the group $\mathfrak{G}_{\mathbb{C}}^{(1)} \times \mathfrak{G}_{\mathbb{C}}^{(2)}$.

2. In $\mathcal{E} x t\left(E_{1}, E_{2}\right)$ there are two equivalence relations to consider: We say that two extension $\mathcal{E}$ and $\mathcal{E}^{\prime}$ in $\mathcal{E} x t\left(E_{2}, E_{1}\right)$ are weakly equivalent, denoted by $\mathcal{E} \sim \mathcal{E}^{\prime}$ if there is a commutative diagram

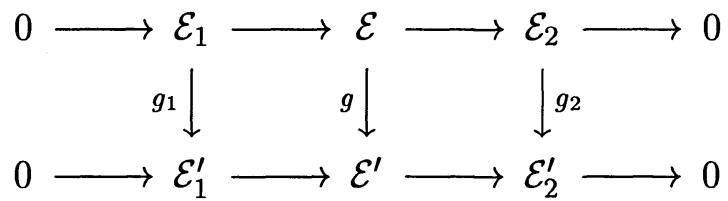

where $g_{1}, g_{2}$, and $g$ are bundle automorphisms of the underlying smooth bundles.

We say that $\mathcal{E}$ and $\mathcal{E}^{\prime}$ are strongly equivalent, denoted by $\mathcal{E} \approx \mathcal{E}^{\prime}$ if $\mathcal{E}_{i}=\mathcal{E}_{i}^{\prime}$ for $i=1,2$, and there is a commutative diagram

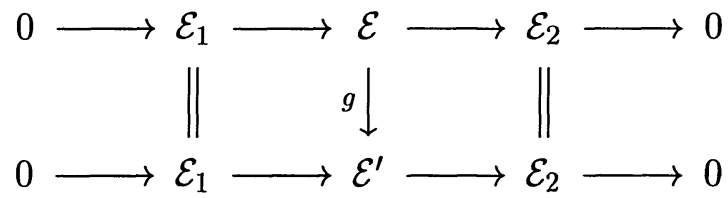

where $g$ is a bundle automorphism of $E$.

3. We can similarly define weak and strong equivalence for surjective triples: Let $\left(\mathcal{E}_{2}, \mathcal{E}, \pi\right)$ and $\left(\mathcal{E}_{2}^{\prime}, \mathcal{E}^{\prime}, \pi^{\prime}\right)$ be surjective triples in $\mathcal{H}_{s}\left(E_{2}, E\right)$. We say that $\left(\mathcal{E}_{2}, \mathcal{E}, \pi\right)$ and $\left(\mathcal{E}_{2}^{\prime}, \mathcal{E}^{\prime}, \pi^{\prime}\right)$ are weakly equivalent, denoted by $\left(\mathcal{E}_{2}, \mathcal{E}, \pi\right) \sim\left(\mathcal{E}_{2}^{\prime}, \mathcal{E}^{\prime}, \pi^{\prime}\right)$, if there is a commutative diagram

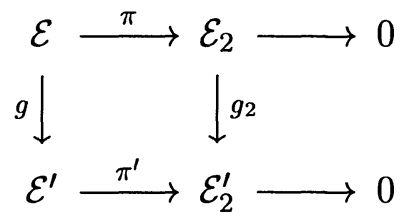

where $g_{2}$ and $g$ are bundle automorphisms of the underlying smooth bundles.

We say that $\left(\mathcal{E}_{2}, \mathcal{E}, \pi\right)$ and $\left(\mathcal{E}_{2}^{\prime}, \mathcal{E}^{\prime}, \pi^{\prime}\right)$ are strongly equivalent, denoted by $\left(\mathcal{E}_{2}, \mathcal{E}, \pi\right) \approx\left(\mathcal{E}_{2}^{\prime}, \mathcal{E}^{\prime}, \pi^{\prime}\right)$,if there is a commutative diagram

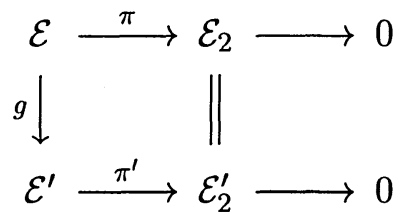


where $g$ is a bundle automorphism of $E$.

The relationships between these spaces can be seen as follows. With $\mathcal{Z}^{(1)}\left(E_{1}, E_{2}\right)$ as in (2.1.2), we have a map

$$
f: \mathcal{Z}^{(1)}\left(E_{1}, E_{2}\right) \longrightarrow \mathcal{E} x t\left(E_{1}, E_{2}\right) .
$$

Indeed, it is clear that given an element $\left(\bar{\partial}_{1}, \bar{\partial}_{2}, \phi\right) \in \mathcal{Z}^{(1)}\left(E_{1}, E_{2}\right)$ we can define a $\bar{\partial}$-operator on $E=E_{1} \oplus E_{2}$ by

$$
\bar{\partial}_{E}=\left(\begin{array}{cc}
\bar{\partial}_{1} & \phi \\
0 & \bar{\partial}_{2}
\end{array}\right) .
$$

This in turn defines an element in $\mathcal{E} x t\left(E_{1}, E_{2}\right)$. Conversely, given an element

$$
0 \longrightarrow \mathcal{E}_{1} \longrightarrow \mathcal{E} \longrightarrow \mathcal{E}_{2} \longrightarrow 0
$$

in $\mathcal{E} x t\left(E_{1}, E_{2}\right)$, by choosing a metric on $\mathcal{E}$ we can identify the smooth underlying bundle to $\mathcal{E}$ with $E_{1} \oplus E_{2}$, and in this way we can define an inverse to (4.1.1). This does however depends on the choice of the metric. In order to get a metric-independent map, we need to consider the image in $\mathcal{H}^{(1)}$, rather than in $\mathcal{Z}^{(1)}$. This is because two different metrics on $\mathcal{E}$ define second fundamental forms $\phi$ and $\phi^{\prime}$ that are related by $\phi^{\prime}=\phi+\bar{\partial}_{1,2} \alpha$ for $\alpha \in \Omega^{0}\left(\operatorname{Hom}\left(E_{2}, E_{1}\right)\right)$. Moreover this map induces a bijection

$$
\frac{\mathcal{E} x t\left(E_{1}, E_{2}\right)}{\approx} \longleftrightarrow \mathcal{H}^{(1)}\left(E_{1}, E_{2}\right)
$$

where $\approx$ denotes strong equivalence.

Similarly, by identifying $\mathcal{E}_{1}$ with $\operatorname{Ker}(\pi)$, we see that there is a bijective correspondence between extensions in $\mathcal{E} x t\left(E_{1}, E_{2}\right)$ and surjective triples in $\mathcal{H}_{s}\left(E_{2}, E\right)$. Furthermore, this correspondence holds at the level of weak or strong equivalence classes.

Let $\mathfrak{G}_{\mathbb{C}}^{(1)}$ and $\mathfrak{G}_{\mathbb{C}}^{(2)}$ be the complex gauge groups of $E_{1}$ and $E_{2}$ respectively. It is clear that these maps descend to the quotients and we obtain

Proposition 4.3. There are one-to-one correspondences

$$
\frac{\mathcal{H}_{s}\left(E_{2}, E\right)}{\sim} \longleftrightarrow \frac{\mathcal{E} x t\left(E_{1}, E_{2}\right)}{\sim} \longleftrightarrow \frac{\mathcal{H}^{(1)}\left(E_{1}, E_{2}\right)}{\mathfrak{G}_{\mathbb{C}}^{(1)} \times \mathfrak{G}_{\mathbb{C}}^{(2)}}
$$


When we speak of a moduli space of extensions supported by the smooth bundles $E_{1}$ and $E_{2}$, it is the quotient $\frac{\mathcal{E x t}\left(E_{1}, E_{2}\right)}{\sim}$ that we have in mind. We will denote equivalence classes in each of these quotients by square brackets, thus for example, $\left[\mathcal{E}_{1}, \mathcal{E}_{2}, \Phi\right]$ is a class in $\frac{\mathcal{H}^{(1)}\left(E_{1}, E_{2}\right)}{\mathfrak{G}_{\mathbb{C}}^{(1)} \times \mathfrak{G}_{\mathbb{C}}^{(2)}}$.

\subsection{Stability.}

In view of the above bijections, it makes sense to compare the stability properties of the surjective triples, of the 1-cohomology triples, and of the extensions. This comparison is made considerably easier if we formulate the respective notions of stability in a uniform way. For this, we use the functions $\theta_{a_{1}, a_{2}, \tau_{1}, \tau_{2}}$ defined earlier.

Recall that for an ordinary triple, the definition of $\tau$-stability in [BGP] is equivalent to $\left(1,1, \tau, \tau^{\prime}\right)$-stability as defined in $\S 2.2$, with $\tau$ and $\tau^{\prime}$ being related by $d_{1}+d_{2}=r_{1} \tau+r_{2} \tau^{\prime}$. Similarly, taking the special values $\left\{a_{1}, a_{2}, \tau_{1}, \tau_{2}\right\}=\left\{1,1, \tau, \tau^{\prime}\right\}$ for a 1-cohomology triple, and defining $\alpha=\tau-\tau^{\prime}$, we get

Definition/Lemma 4.4. The 1-cohomology triple $\left(\mathcal{E}_{1}, \mathcal{E}_{2}, \Phi\right)$ is said to be $\alpha$-stable if for all subtriples, $\left(\mathcal{E}_{1}^{\prime}, \mathcal{E}_{2}^{\prime}, \Phi^{\prime}\right)$, we have

$$
\mu_{\alpha}\left(\mathcal{E}_{1}^{\prime}, \mathcal{E}_{2}^{\prime}\right)<\mu_{\alpha}\left(\mathcal{E}_{1}, \mathcal{E}_{2}\right)
$$

where

$$
\mu_{\alpha}\left(\mathcal{E}_{1}^{\prime}, \mathcal{E}_{2}^{\prime}\right)=\mu\left(\mathcal{E}_{1}^{\prime}, \mathcal{E}_{2}^{\prime}\right)+\alpha \frac{r_{2}^{\prime}}{r_{1}^{\prime}+r_{2}^{\prime}} .
$$

This is equivalent to $(1,1, \tau, \tau-\alpha)$-stability, as defined in Definition 2.7.

Now let $\left(\mathcal{E}_{2}, \mathcal{E}, \pi\right)$ be a surjective triple corresponding to the 1-cohomology triple $\left(\mathcal{E}_{1}, \mathcal{E}_{2}, \Phi\right)$, i.e. $\left[\mathcal{E}_{2}, \mathcal{E}, \pi\right]=\left[\mathcal{E}_{1}, \mathcal{E}_{2}, \Phi\right]$ under the bijection in Proposition 4.3. If we compare the stability of $\left(\mathcal{E}_{2}, \mathcal{E}, \pi\right)$ and $\left(\mathcal{E}_{1}, \mathcal{E}_{2}, \Phi\right)$, we find that we need to introduce a slightly restricted form of stability for the surjective triple. We will refer to this as surjective stability, with the precise definition as follows:

Definition 4.5. Given a surjective triple $\left(\mathcal{E}_{2}, \mathcal{E}, \pi\right)$, we say that a subtriple $\left(\mathcal{E}_{2}^{\prime}, \mathcal{E}^{\prime}, \pi^{\prime}\right)$ is a surjective subtriple if $\pi: \mathcal{E}^{\prime} \longrightarrow \mathcal{E}_{2}^{\prime}$ is surjective.

Fix real numbers $\left\{b_{1}, b_{2}, \sigma_{1}, \sigma_{2}\right\}$ such that

$$
b_{1} \operatorname{deg}\left(\mathcal{E}_{2}\right)+b_{2} \operatorname{deg}\left(\mathcal{E}_{1}\right)-\sigma_{1} \operatorname{rank}\left(\mathcal{E}_{2}\right)-\sigma_{2} \operatorname{rank}(\mathcal{E})=0,
$$


i.e. such that $\theta_{b_{1}, b_{2}, \sigma_{1}, \sigma_{2}}\left(\mathcal{E}_{2}, \mathcal{E}\right)=0$. We say that the triple is $\left(b_{1}, b_{2}, \sigma_{1}, \sigma_{2}\right)$ surjectively stable if

$$
\theta_{b_{1}, b_{2}, \sigma_{1}, \sigma_{2}}\left(\mathcal{E}_{2}^{\prime}, \mathcal{E}^{\prime}\right)<0
$$

for all surjective subtriples $\left(\mathcal{E}_{2}^{\prime}, \mathcal{E}^{\prime}, \pi^{\prime}\right)$.

Remark. In some cases surjective stability is equivalent to full stability. For example:

Proposition 4.6. If $b_{1}=b_{2}$, and $\sigma_{1}-\sigma_{2}>0$, then $\left(b_{1}, b_{2}, \sigma_{1}, \sigma_{2}\right)$-surjective stability is equivalent to $\left(b_{1}, b_{2}, \sigma_{1}, \sigma_{2}\right)$-stability for a surjective triple.

Proof. It is clear from the definitions that stability implies surjective stability. Conversely, suppose that $\left(\mathcal{E}_{2}, \mathcal{E}, \pi\right)$ is a surjective triple which is not $\left\{b_{1}, b_{2}, \sigma_{1}, \sigma_{2}\right\}$-stable. Let $\left(\mathcal{E}_{2}^{\prime}, \mathcal{E}^{\prime}, \pi^{\prime}\right)$ be a destabilizing subtriple, i.e. suppose that $\left(\mathcal{E}_{2}^{\prime}, \mathcal{E}^{\prime}, \pi^{\prime}\right)$ is a subtriple (not necessarily a surjective subtriple), such that

$$
\theta_{b_{1}, b_{2}, \sigma_{1}, \sigma_{2}}\left(\mathcal{E}_{2}^{\prime}, \mathcal{E}^{\prime}\right) \geq 0 .
$$

Suppose that $\left(\mathcal{E}_{2}^{\prime}, \mathcal{E}^{\prime}, \pi^{\prime}\right)$ is not a surjective subtriple. Let $\pi^{\prime}\left(\mathcal{E}^{\prime}\right)$ be the image of the sheaf map, and denote by $\pi^{-1}\left(\mathcal{E}_{2}^{\prime}\right)$ the subsheaf of $\mathcal{E}$ defined by

$$
0 \longrightarrow \operatorname{Ker}\left(\pi^{\prime}\right) \longrightarrow \pi^{\prime-1}\left(\mathcal{E}_{2}^{\prime}\right) \longrightarrow \mathcal{E}_{2}^{\prime} \longrightarrow 0 .
$$

Then $\left(\pi^{\prime}\left(\mathcal{E}^{\prime}\right), \mathcal{E}^{\prime}, \pi^{\prime}\right)$ and $\left(\mathcal{E}_{2}^{\prime}, \pi^{\prime-1}\left(\mathcal{E}_{2}^{\prime}\right), \pi^{\prime}\right)$ are both surjective subtriples of $\left(\mathcal{E}_{2}, \mathcal{E}, \pi\right)$. We will show that if $\theta_{b_{1}, b_{2}, \sigma_{1}, \sigma_{2}}\left(\mathcal{E}_{2}^{\prime}, \mathcal{E}^{\prime}\right) \geq 0$, then at least one of these two surjective subtriples must likewise be destabilizing.

By their definition, the surjective subtriples lead to the following diagram:

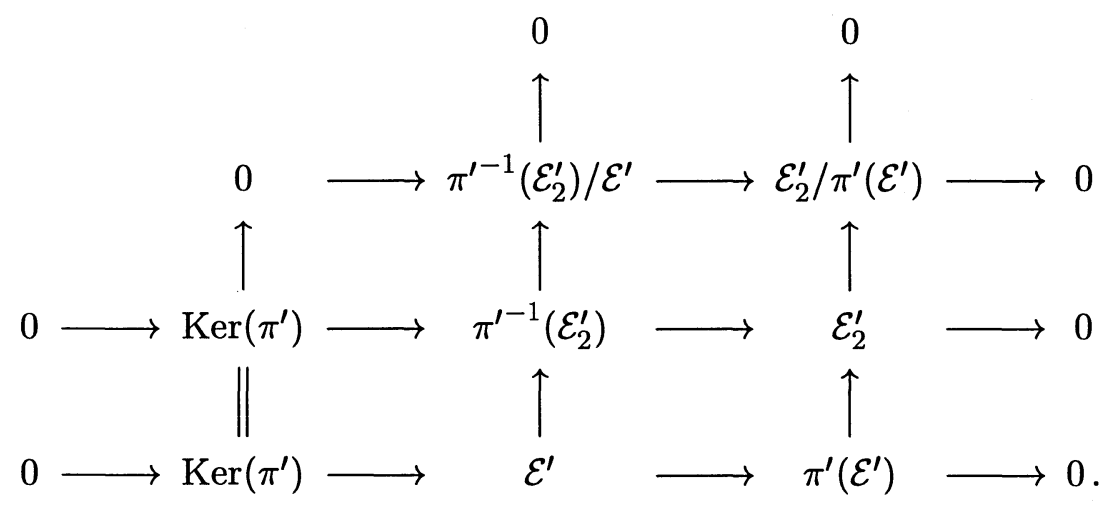


It follows that

$$
\begin{gathered}
\operatorname{deg}\left(\pi^{\prime-1}\left(\mathcal{E}_{2}^{\prime}\right)\right)-\operatorname{deg}\left(\mathcal{E}^{\prime}\right)=\operatorname{deg}\left(\mathcal{E}_{2}^{\prime}\right)-\operatorname{deg}\left(\pi^{\prime}\left(\mathcal{E}^{\prime}\right)\right) \\
\operatorname{rank}\left(\pi^{\prime-1}\left(\mathcal{E}_{2}^{\prime}\right)\right)-\operatorname{rank}\left(\mathcal{E}^{\prime}\right)=\operatorname{rank}\left(\mathcal{E}_{2}^{\prime}\right)-\operatorname{rank}\left(\pi^{\prime}\left(\mathcal{E}^{\prime}\right)\right) .
\end{gathered}
$$

Using this, a computation yields the relation

$$
\begin{aligned}
2 \theta_{b_{1}, b_{2}, \sigma_{1}, \sigma_{2}}\left(\mathcal{E}_{2}^{\prime}, \mathcal{E}^{\prime}\right)= & {\left[\theta_{b_{1}, b_{2}, \sigma_{1}, \sigma_{2}}\left(\pi^{\prime}\left(\mathcal{E}^{\prime}\right), \mathcal{E}^{\prime}\right)+\Theta_{b_{1}, b_{2}, \sigma_{1}, \sigma_{2}}\left(\mathcal{E}_{2}^{\prime}, \pi^{\prime-1}\left(\mathcal{E}_{2}^{\prime}\right)\right)\right]+} \\
& +\left(a_{1}-a_{2}\right) \Delta_{d}+\left(\tau_{2}-\tau_{1}\right) \Delta_{r}
\end{aligned}
$$

where

$$
\begin{gathered}
\Delta_{d}=\operatorname{deg}\left(\pi^{\prime-1}\left(\mathcal{E}_{2}^{\prime}\right)\right)-\operatorname{deg}\left(\mathcal{E}^{\prime}\right), \\
\Delta_{r}=\operatorname{rank}\left(\pi^{\prime-1}\left(\mathcal{E}_{2}^{\prime}\right)\right)-\operatorname{rank}\left(\mathcal{E}^{\prime}\right) .
\end{gathered}
$$

The result follows from this, since $\Delta_{r} \geq 0$.

Remark. In general, this relation between surjective stability and full stability does not seem to be true.

Proposition 4.7. Let $\left(\mathcal{E}_{2}, \mathcal{E}, \pi\right)$ and $\left(\mathcal{E}_{1}, \mathcal{E}_{2}, \Phi\right)$ be related by $\left[\mathcal{E}_{1}, \mathcal{E}_{2}, \Phi\right]=$ $\left[\mathcal{E}_{2}, \mathcal{E}, \pi\right]$ under the bijection of Proposition 4.2. Let $\left\{a_{1}, a_{2}, \tau_{1}, \tau_{2}\right\}$ be any set of real number such that $a_{1} d_{1}+a_{2} d_{2}-\tau_{1} r_{1}-\tau_{2} r_{2}=0$. Then the following are equivalent

1. The 1-cohomology triple $\left(\mathcal{E}_{1}, \mathcal{E}_{2}, \Phi\right)$ is $\left(a_{1}, a_{2}, \tau_{1}, \tau_{2}\right)$-stable,

2. The surjective triple $\left(\mathcal{E}_{2}, \mathcal{E}, \pi\right)$ is $\left(a_{2}-a_{1}, a_{1}, \tau_{2}-\tau_{1}, \tau_{1},\right)$-surjectively stable.

Proof. The proof of this Proposition depends on the following lemma, which describes the relation between the subobjects of $\left(\mathcal{E}_{2}, \mathcal{E}, \pi\right)$ and $\left(\mathcal{E}_{1}, \mathcal{E}_{2}, \Phi\right)$.

Lemma 4.8. Let $\left(\mathcal{E}_{2}, \mathcal{E}, \pi\right)$ and $\left(\mathcal{E}_{1}, \mathcal{E}_{2}, \Phi\right)$ be related by $\left(\mathcal{E}_{1}, \mathcal{E}_{2}, \Phi\right)=$ $f\left(\mathcal{E}_{2}, \mathcal{E}, \pi\right)$. Denote the sets of subobjects of $\left(\mathcal{E}_{2}, \mathcal{E}, \pi\right)$ and $\left(\mathcal{E}_{1}, \mathcal{E}_{2}, \Phi\right)$ by $\mathcal{S U B}\left(\mathcal{E}_{2}, \mathcal{E}, \pi\right)$ and $\mathcal{S U B}\left(\mathcal{E}_{1}, \mathcal{E}_{2}, \Phi\right)$ respectively. Then

1. There is a well defined map

$$
f: \operatorname{SUB}\left(\mathcal{E}_{2}, \mathcal{E}, \pi\right) \longrightarrow \mathcal{S U B}\left(\mathcal{E}_{1}, \mathcal{E}_{2}, \Phi\right)
$$


2. this map is surjective,

3. the function $\theta_{a_{1}, a_{2}, \tau_{1}, \tau_{2}}$ is constant on the fibers of this map.

Proof. (1) Let $\left(\mathcal{E}_{2}^{\prime}, \mathcal{E}^{\prime}, \pi^{\prime}\right)$ be a subtriple of $\left(\mathcal{E}_{2}, \mathcal{E}, \pi\right)$, and let $\Phi^{\prime}$ be the extension class of the extension

$$
0 \longrightarrow \operatorname{Ker}\left(\pi^{\prime}\right) \longrightarrow \mathcal{E}^{\prime} \longrightarrow \mathcal{E}_{2}^{\prime} \longrightarrow 0
$$

We need to check that $\left(\operatorname{Ker}\left(\pi^{\prime}\right), \mathcal{E}_{2}^{\prime}, \Phi^{\prime}\right)$ is in $\mathcal{S U B}\left(\mathcal{E}_{1}, \mathcal{E}_{2}, \Phi\right)$. But this is an immediate consequence of the way in which subobjects are defined. We can thus define $f\left(\mathcal{E}_{2}^{\prime}, \mathcal{E}^{\prime}, \pi^{\prime}\right)=\left(\operatorname{Ker}\left(\pi^{\prime}\right), \mathcal{E}_{2}^{\prime}, \Phi^{\prime}\right)$.

(2) If $\left(\mathcal{E}_{1}^{\prime}, \mathcal{E}_{2}^{\prime}, \Phi^{\prime}\right)$ is a subobject of $\left(\mathcal{E}_{1}, \mathcal{E}_{2}, \Phi\right)$, then $\mathcal{E}_{2}^{\prime}$ is a subbundle of $\mathcal{E}_{2}$. Furthermore, if $\left(\mathcal{E}_{1}, \mathcal{E}_{2}, \Phi\right)=f\left(\mathcal{E}_{2}, \mathcal{E}, \pi\right)$, then (again, by the defining properties of subobjects) $\mathcal{E}_{2}^{\prime}$ can be lifted to a subbundle $\mathcal{E}^{\prime} \subset \mathcal{E}$. Then, with $\pi^{\prime}: \mathcal{E}^{\prime} \longrightarrow \mathcal{E}_{2}^{\prime}$ denoting the projection map, $\left(\mathcal{E}_{2}^{\prime}, \mathcal{E}^{\prime}, \pi^{\prime}\right)$ is in $\mathcal{S U} \mathcal{B}\left(\mathcal{E}_{2}, \mathcal{E}, \pi\right)$. Thus the map $f$ is surjective.

(3) This is clear since all subtriples $\left(\mathcal{E}_{2}^{\prime}, \mathcal{E}^{\prime}, \pi^{\prime}\right)$ in $f^{-1}\left(\mathcal{E}_{1}^{\prime}, \mathcal{E}_{2}^{\prime}, \Phi^{\prime}\right)$ have isomorphic underlying smooth bundles.

The proof of the proposition now follows from a straightforward computation. Given subobjects related by $\left(\mathcal{E}_{2}^{\prime}, \mathcal{E}^{\prime}, \pi^{\prime}\right)=f\left(\mathcal{E}_{1}^{\prime}, \mathcal{E}_{2}^{\prime}, \Phi^{\prime}\right)$, we get

$$
\begin{aligned}
\Theta_{a_{1}, a_{2}, \tau_{1}, \tau_{2}}\left(\mathcal{E}_{1}^{\prime}, \mathcal{E}_{2}^{\prime}\right) & =a_{1} d_{1}^{\prime}+a_{2} d_{2}^{\prime}-\tau_{1} r_{1}^{\prime}-\tau_{2} r_{2}^{\prime} \\
& =a_{1}\left(d^{\prime}-d_{2}^{\prime}\right)+a_{2} d_{2}^{\prime}-\tau_{1}\left(r^{\prime}-r_{2}^{\prime}\right)-\tau_{2} r_{2}^{\prime} \\
& =a_{1} d^{\prime}+\left(a_{2}-a_{1}\right) d_{2}^{\prime}-\tau_{1} r^{\prime}-\left(\tau_{2}-\tau_{1}\right) r_{2}^{\prime} \\
& =\Theta_{a_{2}-a_{1}, a_{1}, \tau_{2}-\tau_{1}, \tau_{1}}\left(\mathcal{E}_{2}^{\prime}, \mathcal{E}^{\prime}\right) .
\end{aligned}
$$

Given an extension

$$
0 \longrightarrow \mathcal{E}_{1} \longrightarrow \mathcal{E} \longrightarrow \mathcal{E}_{2} \longrightarrow 0
$$

the strong equivalence class of $e$ can be identified, as we have seen above, with the 1-cohomology triple $T=\left(\mathcal{E}_{1}, \mathcal{E}_{2}, \Phi\right)$, where $\Phi \in H^{1}\left(\mathcal{E}_{1} \otimes \mathcal{E}_{2}^{*}\right)$ is the class defined by $e$. The weak equivalence class, denoted by $[e]$ corresponds to the equivalence class of $T$ in $\mathcal{H}^{(1)}\left(E_{1}, E_{2}\right) / \mathfrak{G}_{\mathbb{C}}^{(1)} \times \mathfrak{G}_{\mathbb{C}}^{(2)}$. By a comparison of the appropriate subobjects it is apparent that the stability notions we have defined for extensions is a property of weak equivalence classes. Similarly, stability of 1-cohomology triples is a property of equivalence classes under the action of $\mathfrak{G}_{\mathbb{C}}^{(1)} \times \mathfrak{G}_{\mathbb{C}}^{(2)}$. In other words 
Lemma 4.9. 1. An extension e is $\alpha$-stable if and only if every extension $e^{\prime}$ such that $[e]=\left[e^{\prime}\right]$ is $\alpha$-stable.

2. A 1-cohomology triple $T$ is $\alpha$-stable if and only if every triple $T^{\prime}$ such that $\left[T^{\prime}\right]=[T]$ is $\alpha$-stable.

Proposition 4.10. Let $[e]$ be a class in $\mathcal{E} x t\left(E_{1}, E_{2}\right) / \sim$ and let $[T]$ be the corresponding equivalence class in $\mathcal{H}^{(1)}\left(E_{1}, E_{2}\right) / \mathfrak{G}_{\mathbb{C}}^{(1)} \times \mathfrak{G}_{\mathbb{C}}^{(2)}$. Then $[e]$ is $\alpha$-stable if and only if $[T]$ is $\alpha$-stable.

Proof. Again, the proof depends on a comparison of subobjects. Suppose, for example, that for some $e \in[e]$ there is a subextension which violates the $\alpha$-stability condition. But this subextension determines a sub-triple of $T(e)$, the 1-cohomology triple corresponding to $e$, and this subtriple violates the $\alpha$-stability condition for $T(e)$. Conversely, suppose one is given a 1-cohomology triple $T$, and a subtriple $T^{\prime}$ which violates stability. This subtriple determines a subextension for some extension, say $e(T)$, in the class corresponding to $T$, and this subextension violates the $\alpha$-stability condition for $e(T)$.

Combining Definition/Lemma 4.4, Proposition 4.7, and Proposition 4.10, we thus get

Proposition 4.11. Let the extension $0 \longrightarrow \mathcal{E}_{1} \longrightarrow \mathcal{E} \longrightarrow \mathcal{E}_{2} \longrightarrow 0$, the surjective triple $\left(\mathcal{E}_{2}, \mathcal{E}, \pi\right)$, and the 1-cohomology triple $\left(\mathcal{E}_{1}, \mathcal{E}_{2}, \Phi\right)$ be related as described above. Then the following are equivalent

1. the extension $0 \longrightarrow \mathcal{E}_{1} \longrightarrow \mathcal{E} \longrightarrow \mathcal{E}_{2} \longrightarrow 0$ is $\alpha$-stable,

2. the 1-cohomology triple $\left(\mathcal{E}_{1}, \mathcal{E}_{2}, \Phi\right)$ is $\alpha$-stable,

3. the 1-cohomology triple $\left(\mathcal{E}_{1}, \mathcal{E}_{2}, \Phi\right)$ is $(1,1, \tau, \tau-\alpha)$-stable,

4. the surjective triple $\left(\mathcal{E}_{2}, \mathcal{E}, \pi\right)$ is $(0,1,-\alpha, \tau)$-stable.

In (3) and (4), $\tau$ is determined by the relation $d_{1}+d_{2}=r_{1} \tau+r_{2}(\tau-\alpha)$.

\subsection{Metric equations.}

Corresponding to the comparison between the stability properties of surjective triples, 1-cohomology triples and extensions, there is an analogous 
comparison between the equations governing the metric problems in the three situations. In this section we spell out this equivalence of metric problems.

For the 1-cohomology triple $\left(\mathcal{E}_{1}, \mathcal{E}_{2}, \Phi\right)$, the equations corresponding to $\left(a_{1}, a_{2}, \tau_{1}, \tau_{2}\right)$-stability are given by $(2.4 .7 \mathrm{a}-\mathrm{c})$, i.e.

$$
\begin{gathered}
i \Lambda a_{1} F_{H_{1}}+\Lambda^{n}\left(\phi \circ \bar{*}_{E} \phi\right)=\tau_{1} \mathbf{I}_{1}, \\
i \Lambda a_{2} F_{H_{2}}-(-1)^{p} \Lambda^{n}\left(\bar{*}_{E} \phi \circ \phi\right)=\tau_{2} \mathbf{I}_{2}, \\
\bar{\partial}_{1,2}^{*}(\phi)=0,
\end{gathered}
$$

where $\phi \in \Omega^{0, p}\left(X, \operatorname{Hom}\left(E_{2}, E_{1}\right)\right)$ is a representative of the cohomology class $\Phi$.

The equations corresponding to, say, $\left(b_{1}, b_{2}, \sigma_{1}, \sigma_{2}\right)$ - stability for a (surjective) triple $\left(\mathcal{E}_{2}, \mathcal{E}, \pi\right)$, come from (2.4.7) with $p=0$. They are

$$
\begin{gathered}
b_{1} i \Lambda F_{H_{2}}+\pi \pi^{*}=\sigma_{1} \mathbf{I}_{2} \\
b_{2} i \Lambda F_{H}-\pi^{*} \pi=\sigma_{2} \mathbf{I} .
\end{gathered}
$$

Proposition 4.11. Let $\left(\mathcal{E}_{1}, \mathcal{E}_{2}, \Phi\right)$ be a 1-cohomology triple, and let $\left(\mathcal{E}_{2}, \mathcal{E}, \pi\right)$ be a corresponding surjective triple. Suppose that there are metrics $\mathrm{H}_{2}$ and $\mathrm{H}$ on $\left(\mathcal{E}_{2}, \mathcal{E}, \pi\right)$ satisfying (4.3.1a,b).

Then there are metrics $H_{1}$ and $H_{2}$ and a representative $\phi \in \Phi$ satisfying (2.4.7) with parameters $\left(b_{2}, b_{2}+b_{1}, \sigma_{2}, \sigma_{1}+\sigma_{2}\right)$.

Proof. We can use the metric on $E$ to fix an orthogonal decomposition $E=E_{1} \oplus E_{2}$. Let $\phi$ be the element in $\Omega^{0, p}\left(X, \operatorname{Hom}\left(E_{2}, E_{1}\right)\right)$ corresponding to the second fundamental form with respect to this metric. Then

$$
F_{H}=\left(\begin{array}{cc}
F_{H_{1}}-\phi \wedge \phi^{*} & D_{1,2}^{\prime} \phi \\
-\bar{\partial}_{1,2} \phi^{*} & F_{H_{2}}-\phi^{*} \wedge \phi
\end{array}\right)
$$

where $D_{1,2}^{\prime}$ is the holomorphic part of the metric connection on $\operatorname{Hom}\left(E_{2}, E_{1}\right)$. Furthermore, in this frame, we get

$$
\pi^{*} \pi=\left(\begin{array}{cc}
0 & 0 \\
0 & \pi \pi^{*}
\end{array}\right)
$$


Equation (4.3.1b) thus decomposes as

$$
\begin{aligned}
\bar{\partial}_{1,2}^{*} \phi & =0, \\
i \Lambda b_{2} F_{H_{1}}-i b_{2} \Lambda\left(\phi \wedge \phi^{*}\right) & =\sigma_{2} \mathbf{I}_{1}, \\
i \Lambda b_{2} F_{H_{2}}-i b_{2} \Lambda\left(\phi^{*} \wedge \phi\right) & =\sigma_{2} \mathbf{I}_{2}+\pi \pi^{*} .
\end{aligned}
$$

Since $\phi$ is in $\Omega^{0,1}\left(X, \operatorname{Hom}\left(E_{2}, E_{1}\right)\right)$, i.e. has form degree $(0,1)$, we get

$$
-i \Lambda\left(\phi \wedge \phi^{*}\right)=\frac{1}{n !} \Lambda^{n}\left(\phi \circ \bar{*}_{E} \phi\right) \text { and }-i \Lambda\left(\phi^{*} \wedge \phi\right)=-\frac{1}{n !} \Lambda^{n}\left(\bar{*}_{E} \phi \circ \phi\right) \text {. }
$$

Combining (4.3.3) with (4.3.1), we thus get

$$
\begin{gathered}
i \Lambda b_{2} F_{H_{1}}+\frac{b_{2}}{n !} \Lambda^{n}\left(\phi \circ \bar{*}_{E} \phi\right)=\sigma_{2} \mathbf{I}_{1}, \\
i \Lambda\left(b_{2}+b_{1}\right) F_{H_{2}}+\frac{b_{2}}{n !} \Lambda^{n}\left(\bar{*}_{E} \phi \circ \phi\right)=\left(\sigma_{2}+\sigma_{1}\right) \mathbf{I}_{2}, \\
\bar{\partial}_{1,2}^{*}(\phi)=0 .
\end{gathered}
$$

The factor $\frac{b_{2}}{n !}$ can be absorbed by rescaling the metric on $E_{1}$. We thus recover the 1-cohomology equations if we set $a_{1}=b_{2}, a_{2}=b_{1}+b_{2}, \tau_{1}=$ $\sigma_{2}, \tau_{2}=\sigma_{1}+\sigma_{2}$.

In the special case where $\left(b_{1}, b_{2}, \sigma_{1}, \sigma_{2}\right)=\left(0,1, \tau, \tau^{\prime}\right)$, the correspondence between these equations and the deformation of the Hermitian-Einstein equation given in (3.2.1) can be seen as follows.

Notice first what happens to the surjective triples equations (4.3.1) in the special case where we take $\left(a_{1}, a_{2}, \tau_{1}, \tau_{2}\right)=(0,1,-\alpha, \tau)$. Denoting the triple by $\left(\mathcal{E}_{2}, \mathcal{E}, \pi\right)$, the metric equations become

$$
\begin{gathered}
\pi \pi^{*}=-\alpha \mathbf{I}_{2}, \\
i \Lambda F_{H}-\pi^{*} \pi=\tau \mathbf{I} .
\end{gathered}
$$

The first of these equations says that $(-\alpha)^{-1} \pi^{*}$ is a left inverse of $\pi$, i.e. that $(-\alpha)^{-1} \pi^{*}$ splits the sequence

$$
0 \longrightarrow \operatorname{Ker}(\pi) \longrightarrow \mathcal{E} \longrightarrow \mathcal{E}_{2} \longrightarrow 0 \text {. }
$$

With respect to the smooth splitting $E=\operatorname{Ker}(\pi) \oplus \pi^{*}\left(E_{2}\right)$, the endomorphism $\pi^{*} \pi$ thus has the block decomposition

$$
\pi^{*} \pi=\left(\begin{array}{cc}
0 & 0 \\
0 & -\alpha \mathbf{I}_{2}
\end{array}\right) .
$$


With $\tau^{\prime}$ defined by $\alpha=\tau-\tau^{\prime}$, the equation (4.3.4b) can then be rewritten as

$$
i \Lambda F_{H}=\left(\begin{array}{cc}
\tau \mathbf{I}_{1} & 0 \\
0 & \tau^{\prime} \mathbf{I}_{2}
\end{array}\right),
$$

which is precisely equation (3.2.1). On the other hand for the 1-cohomology triple $T=\left(\mathcal{E}_{1}, \mathcal{E}_{2}, \Phi\right)$, the equations become

$$
\begin{aligned}
\bar{\partial}_{1,2}^{*} \phi & =0 \\
i \Lambda\left(F_{H_{1}}-\phi \wedge \phi^{*}\right) & =\tau_{1} \mathbf{I}_{1} \\
i \Lambda\left(F_{H_{2}}-\phi^{*} \wedge \phi\right. & =\tau_{2} \mathbf{I}_{2}
\end{aligned}
$$

for a triple $\left(H_{1}, H_{2}, \phi\right)$ consisting of metrics on $\mathcal{E}_{1}$ and $\mathcal{E}_{2}$ respectively, and $\phi \in \mathbf{O}(\Phi)$, where

$$
\mathbf{O}(\Phi)=\left\{\phi \in \Omega^{0,1}\left(\operatorname{Hom}\left(E_{2}, E_{1}\right)\right) \mid \bar{\partial}_{1,2}^{*} \phi=0 \text { and }[\phi]=\Phi\right\} .
$$

The equivalence of these equations with (3.2.1) follows immediately from writing $F_{H}$ as in (4.3.2), and from the fact that $i \Lambda \partial_{1,2}=\bar{\partial}_{1,2}^{*}$.

To have a complete equivalence between the solution of the two metric problems we need to prove the following.

Lemma 4.12. There is a one-to-one correspondence

$$
\operatorname{Met}(\mathcal{E}) \longleftrightarrow \operatorname{Met}\left(\mathcal{E}_{1}\right) \times \operatorname{Met}\left(\mathcal{E}_{2}\right) \times \mathbf{O}(\Phi)
$$

Proof. We have already mentioned above how from a metric $H$ on $\mathcal{E}$ we obtain $\left(H_{1}, H_{2}, \phi\right)$. To prove the other direction we observe that giving a metric on $\mathcal{E}$ is equivalent to giving metrics on $\mathcal{E}_{1}$ and $\mathcal{E}_{2}$ and a $C^{\infty}$-splitting of $E$. But there is a one-to-one correspondence between $C^{\infty}$-splittings of $E$ and elements of $\mathbf{O}(\Phi)$. These is clear since two different splittings $\gamma_{1}, \gamma_{2}$ : $\mathcal{E}_{2} \longrightarrow \mathcal{E}$ differ by an element $\alpha \in \Omega^{0}\left(\operatorname{Hom}\left(\mathcal{E}_{2}, \mathcal{E}_{1}\right)\right)$, i.e. $\gamma_{2}=\gamma_{1}+j \alpha$, where $j$ denotes the inclusion $\mathcal{E}_{1} \longrightarrow \mathcal{E}$. The corresponding fundamental forms are related by $\phi_{2}=\phi_{1}+\bar{\partial} \alpha$.

Summarizing the results for these special values of the parameters $\left(a_{1}, a_{2}, \tau_{1}, \tau_{2}\right)$, we get the following analog of Proposition 4.11:

Proposition 4.13. Let the extension $0 \longrightarrow \mathcal{E}_{1} \longrightarrow \mathcal{E} \longrightarrow \mathcal{E}_{2} \longrightarrow 0$, the surjective triple $\left(\mathcal{E}_{2}, \mathcal{E}, \pi\right)$, and the 1-cohomology triple $\left(\mathcal{E}_{1}, \mathcal{E}_{2}, \Phi\right)$ be related as described above. Then the following are equivalent 
1. The surjective triple $\left(\mathcal{E}_{2}, \mathcal{E}, \pi\right)$ admits a solution (i.e. metrics on $E$ and $E_{2}$ ) to the equations (4.3.1) with $b_{1}=0, b_{2}=1, \sigma_{1}=-\alpha, \sigma_{2}=\tau$.

2. The 1-cohomology triple admits a solution (i.e. a representative of $\Phi$ and metrics on $E_{1}$ and $E_{2}$ ) to the equations (2.4.7) with $a_{1}=a_{2}=$ $1, \tau_{1}=\tau$, and $\tau_{2}=\tau-\alpha$.

3. The bundle $\mathcal{E}$ admits a solution (i.e. a metric on $E$ ) to the equation (3.2.1) with right hand side $\left(\begin{array}{cc}\tau \mathbf{I}_{1} & 0 \\ 0 & (\tau-\alpha) \mathbf{I}_{2}\end{array}\right)$.

\section{References.}

[B] Bradlow, S.B. Special metrics and stability for holomorphic bundles with global sections, J. Diff. Geom. 33 (1991), 169-214.

[BDGW] Bradlow, S.B., Daskalopoulos, G., García-Prada, O., and Wentworth, R. Augmented bundles over Riemann surfaces, Proceedings of 1993 LMS Durham Symposium on Vector Bundles and Algebraic Geometry, CUP 1995.

[BGP] Bradlow, S.B. and García-Prada, O. Stable triples, equivariant bundles and dimensional reduction, Math. Ann. (to appear).

[Do1] Donaldson, S. Anti-self-dual Yang-Mills connections on a complex algebraic surface and stable vector bundles, Proc. Lond. Math. Soc. 50 (1985), 1-26.

[Do2] Donaldson, S. Infinite determinants, stable bundles and curvature, Duke Math. J. 3 (1987), 231-247.

[DUW] Daskalopoulos, G., Uhlenbeck, K., and Wentworth, R. Moduli of extensions of holomorphic bundles on Kähler manifolds, preprint.

[GP] García-Prada, O. Dimensional reduction of stable bundles, vortices and stable pairs, Int. J. Math. 5 (1994), 1-52.

[G] Gunning, R.C. Lectures on vector bundles over Riemann surfaces, Math. Notes, Princeton University Press, 1967.

[Ko] Kobayashi, S. Differential Geometry of Complex Vector Bundles, Princeton University Press, 1987. 
[L] Lübke, M. Stability of Einstein-Hermitian vector bundles, Manuscripta Mathematica, 42 (1983), 245-257.

[S] Simpson, C. Constructing variations of Hodge structure using Yang-Mills theory, JAMS, 1 (1988).

[PT] Pidstrigach, V. and Tyurin, A. Invariants of the smooth structures of an algebraic surfaces arising from Dirac operator, Iz. AN SSSR, 52:2 (1992), 279-371, Russian; English transl. in Warwick preprint, 1992.

[UY] Uhlenbeck, K.K. and Yau, S.T. On the existence of HermitianYang-Mills connections in stable vector bundles, Comm. Pure. Appl. Math. 39 (1986), 5257-5293.

[W] Wells, R.O. Differential Analysis on Complex Manifolds, SpringerVerlag, 1980.

[Wi] Witten, E. Monopoles and four-manifolds, Math. Res. Lett. 1 (1994), 769-796.

RECEIVED JuLY 5Th, 1995.

DEPARTMENT OF MATHEMATICS

UNIVERSITY OF ILLINOIS

URBANA, IL 61801

E-MAIL ADDRESS: BRADLOW@UIUC.EDU

AND

Departamento de Matemáticas

UNIVERSIDAD AUTÓNOMA DE MADRID

28049 MADRID, SPAIN

E-MAIL ADDRESS: OGPRADA@CCUAM3.SDI.UAM.ES 